Too Sweet to Eat:

Exploring the Effects of Cuteness on Meat Consumption

\author{
Janis H. Zickfeld*, Jonas R. Kunst \\ University of Oslo
}

Sigrid M. Hohle

Simula Research Laboratory

In press at Appetite. This paper is not the copy of record and may not exactly replicate the authoritative document published in Appetite.

Author Note

Janis H. Zickfeld and Jonas R. Kunst, Department of Psychology, University of Oslo, Norway; Sigrid M. Hohle, Simula Research Laboratory, Oslo, Norway.

*Correspondence concerning this article should be addressed to Janis H. Zickfeld, Postboks 1094 Blindern, 0317 Oslo, Norway. E-mail: j.h.zickfeld@psykologi.uio.no. 


\begin{abstract}
Although daily meat consumption is a widespread habit, many individuals at the same time put a high value on the welfare of animals. While different psychological mechanisms have been identified to resolve this cognitive tension, such as dissociating the animal from the consumed meat or denying the animal's moral status, few studies have investigated the effects of the animal's appearance on the willingness to consume its meat. The present article explored how the perception of cuteness influences hypothetical meat consumption. We hypothesized that cuter animals would reduce the willingness to consume meat, and that this relationship would be mediated by empathy felt towards the animal. Across four preregistered studies sampling 1074 US and Norwegian participants, we obtained some support for this prediction in the US but to a lesser degree in Norway. However, in all studies an indirect mediation effect of cuteness on meat consumption going through empathy towards the animal was observed. We also explored possible moderating and additional mediating mechanisms of trait pro-social orientation, caretaking intentions and sex effects for which we found mixed evidence. Theoretical and practical implications of the findings are discussed.
\end{abstract} Words: $186 / 280$

Keywords: cuteness, meat consumption, empathy, meat paradox 


\section{Too Sweet to Eat: Exploring the Effects of Cuteness on Meat Consumption}

Many individuals, in general, disapprove of actions harming animals, but simultaneously enjoy meat consumption on a daily basis. Empirical research has put forward a number of theories accounting for this so-called meat paradox (Loughnan, Haslam, \& Bastian, 2010). One way to solve such cognitive dissonance might be denying the moral status or mental capacity of the animal (Bratanova, Loughnan, \& Bastian, 2011; Loughnan et al., 2010). Other arguments have involved nutritional, evolutionary or merely hedonic justifications for meat consumption (Bohm, Lindblom, Åbacka, Bengs, \& Hörnell, 2015; Piazza et al., 2015; Rothgerber, 2013b). Finally, another perspective has argued that consumers often dissociate meat from its animal origins (e.g., Adams, 2015; Rothgerber, 2013a). In fact, recent findings manipulating the context of meat presentation support this idea by highlighting the role of dissociation, empathy and disgust (Kunst \& Hohle, 2016). In one study, the authors varied the display of a lamb in a meat advertisement, resulting in less self-reported willingness to consume the product when the animal was present. This path was mediated by self-reported dissociation and, subsequently, empathy towards the target animal. Yet, as the authors noted, the study was limited because it did not measure an alternative, probable pathway that may lead to lowered hypothetical meat consumption, namely the degree to which consumers perceived the animal displayed in the advertisement as cute.

Cuteness responses are evoked by objects that have infant-like features (so-called Kindchenschema, Lorenz, 1943; e.g., Borgi, Cogliati-Dezza, Brelsford, Meints, \& Cirulli, 2014). Studies have linked cuteness to increased empathy, compassion (Aragón, Clark, Dyer, \& Bargh, 2015; Kringelbach, Stark, Alexander, Bornstein, \& Stein, 2016; Lishner, Oceja, Stocks, \& Zaspel, 2008; Sherman \& Haidt, 2011), and caretaking (Glocker et al., 2009; Keating, Randall, Kendrick, \& Gutshall, 2003; Nittono, Fukushima, Yano, \& Moriya, 2012), arguably highlighting responses to cuteness as adaptive evolution (Leitão \& Castelo-Branco, 
2010; Preston, 2013). Evidence for the relation between cuteness and meat consumption comes from a correlational study in which participants reported more disgust about eating meat from animals that looked cuter than normal (Ruby \& Heine, 2012). However, although a number of attempts have been made to explore the general nature of cuteness responses, no study to date has systematically tested the effect of cuteness on meat consumption. In the present paper, we aimed to fill this gap, exploring the effects of cuteness responses through pathways of empathy, humanization, and caretaking responses using correlational and experimental designs.

Different terms such as cuteness, the cute-emotion, or aww (Buckley, 2016) have been used to refer to a specific perception of, or responses to, infant-like features in the socialscientific literature. A number of studies have experimentally tested whether altering such infant-like aspects results in cuteness perceptions and responses (Borgi et al., 2014; Glocker et al., 2009; Little, 2012). For instance, Little (2012) manipulated human adult or infant faces as well as faces of non-human animals (i.e., cats). Results suggested that infant-like characteristics made both human and animal faces cuter. Another study presented similar evidence using dog and cat stimuli (Borgi et al., 2014). Throughout the manuscript, the term cuteness is used to denote responses to such perceptions of infant-like or baby schema traits of non-human animals. Although some scholars have suggested that the cuteness concept should also include aspects such as infant smells or sounds (Kringelbach et al., 2016), we merely focus on the visual domain here.

The phenomenon of cuteness has been observed across a number of cultures, with some having evolved more profound societal implementations such as the Japanese kawaii, which is roughly translated as cute (Nittono et al., 2012). Moreover, empirical research has identified several inter-individual and biological differences in cuteness responses. First of all, adult participants who had siblings reported more cuteness in response to children's faces 
than those without siblings (Luo, Kendrick, Li, \& Lee, 2015). Further research has pointed at sex differences in perceiving cuteness (Lobmaier, Sprengelmeyer, Wiffen, \& Perrett, 2010; Sprengelmeyer et al., 2009). While participants of both sexes performed similarly in accurately reporting an infant's emotion and age, females were more likely to reliably detect the cuter infant (Lobmaier et al., 2010). These sex differences have been suggested to be based on evolutionary and biological aspects. Providing support for this notion, females reported a better discrimination of cute and non-cute infant faces during ovulation (Lobmaier, Probst, Perrett, \& Heinrichs, 2015). In addition, young women and premenopausal females performed better at detecting cuteness differences than older women or men did (Sprengelmeyer et al., 2009). The authors concluded that female reproductive hormones play a crucial role in cuteness perception. Some research has replicated sex differences regarding cuteness perception, but not its motivational components (i.e., caretaking; Parsons, Young, Kumari, Stein, \& Kringelbach, 2011).

In line with an evolutionary perspective, it has been argued that traits evoking cuteness are vital in conveying neonatal vulnerability (Leitão \& Castelo-Branco, 2010; Preston, 2013). In light of this evolutionary account, it makes sense that cuteness should result in higher empathy with the elicitor and increased attentiveness or nurturing behavior. Taking care and feeling compassion for infants or young children enhances their adaptive fitness and chances for survival. A similar account has been proposed in order to explain empathy or caretaking behavior towards animals (Bradshaw \& Paul, 2010).

A number of studies have addressed the effects of perceiving cuteness and related responses to infant-like traits. Exploring affective, cognitive and motivational aspects, the majority of these studies has identified the importance of empathy, humanization and caretaking behavior. Most studies on cuteness and empathy have defined empathy in the context of empathic concern or sympathy, that is, a positive compassionate or tender response 
to vulnerable targets or others in need (Davis, 1980). Hence, throughout this research, we will refer to these concepts or definitions when employing the term empathy.

Various theories have linked cuteness responses to empathy. For instance, cuteness has been conceptualized as a moral emotion leading to empathy or compassion as part of a moral circle (Sherman \& Haidt, 2011). In a first empirical attempt to test this association, Batson, Lishner, Cook, and Sawyer (2005) investigated whether variation in empathy is mostly due to perceived similarity with the target or the idea of nurturance. Their findings suggested that nurturance (i.e., taking care of vulnerable others) was associated with empathy, while similarity was not. Testing the direct effect of cuteness on empathy, another study manipulated infant-like traits in adult photographs (Lishner et al., 2008). Results suggested that participants reported more empathic concern towards cuter images, providing experimental support for such a relationship.

From a more cognitive viewpoint, Sherman and Haidt (2011) argued that cuteness responses result in humanizing the target: that is, ascribing more human-like traits to them. To date we are not aware of any empirical account trying to test this prediction.

Much emphasis regarding the effects of cuteness has been put on testing consequential motivations, such as caretaking and helping, or cognitive functions including attention allocation (Sherman, Haidt, \& Coan, 2009). A number of theoretical accounts have argued that such motivations are direct and causal outcomes of cuteness responses (Kringelbach et al., 2016; Sherman \& Haidt, 2011). Extensive experimental evidence has been provided on this proposition (Glocker et al., 2009; Nittono et al., 2012; Sherman et al., 2009). In one study, viewing cute puppies or kittens in contrast to more neutral cats and dogs led participants to act more carefully, making fewer errors in a fine motor task (Sherman et al., 2009). In a similar paradigm, the effects of cuteness responses on physical care in a precision task were moderated by the pro-social orientation of female participants (Sherman, 
Haidt, Iyer, \& Coan, 2013). Specifically, female participants scoring high on a pro-social orientation measure showed more physical care by making fewer errors in the task after watching cute stimuli than less pro-social participants did. Similar results were observed with Japanese participants, suggesting some cross-cultural validity of the relationship (Nittono et al., 2012). Further evidence is provided by an experimental study employing cute and noncute infant pictures and assessing motivations to take care of these infants (Glocker et al., 2009). Participants viewing cute infants reported increased intentions to take care of them compared to those viewing non-cute faces. Finally, one study with high ecologic validity used the 'lost letter technique' to test the effects of cuteness on helping behavior (Keating et al., 2003). Stamped fictional resumes depicting cute or non-cute European or African American male or female adults were distributed in the US and Kenya. Results indicated that resumes including cute European and African American females were posted more often than their non-cute counterparts. The same effect was observed for European American males, but not for African American males. Posting of the resumes was an operationalization of helping behavior by delivering the resume on behalf of the fictional candidate.

While an emerging body of research has looked at the behavioral and motivational consequences of cuteness for human-human relationships, some studies have also investigated its effects on behavior towards domestic animals such as cats or dogs. Given the topic of the present research, we will now narrow our focus to the association between cuteness and meat consumption, and also elaborate on the potential importance of empathy, humanization and caretaking.

Few investigations have attempted to directly explore the relationship between cuteness responses and meat consumption. One of the few thorough attempts was provided by Grauerholz (2007). Theorizing that the meat paradox — consuming meat, but still valuing animal welfare - is affected by consumers' differentiation between meat and their animal 
origins, the author conducted a qualitative analysis of advertisements. She concluded that the dissociation between meat and animals is actually potentiated by an objectification or 'cutification' of the animal, which distracts attention away from meat's animal origins. However, this account stands in stark contrast to the general literature on cuteness responses, which highlights its role as a factor increasing empathic and caretaking responses. For instance, in a cross-cultural survey assessing factors influencing meat consumption, participants reported increased disgust and less willingness to consume meat from animals that deviated from a neutral appearance by either being particularly ugly or cute (Ruby \& Heine, 2012). These correlational findings suggest that cuteness might play an important role in reducing willingness to consume meat.

Past research has also indicated that the perceived mental capacity or humanness of animals might influence meat consumption. For instance, participants attributed less mental capacity for suffering to animals after consuming dried beef in contrast to nuts (Loughnan et al., 2010). Correlational evidence has also linked intelligence or mental capacity of the animal to disgust and willingness to consume meat (Ruby \& Heine, 2012). As some scholars have suggested a link between cuteness and humanization (Sherman \& Haidt, 2011), perceived cuteness might result in ascribing more sophisticated mental capacities to the animal, thereby decreasing willingness to consume meat. However, recent studies were not able to show an effect of mental capacity on willingness to eat meat when controlling for other variables such as disgust or empathy (Kunst \& Hohle, 2016).

Indeed, another factor important for meat consumption seems to be empathy towards the animal (Cerjak, Karolyi, \& Mesić, 2011; Rothgerber \& Mican, 2014). Across a number of studies, Kunst and Hohle (2016) observed that empathy towards the slaughtered animal negatively predicted willingness to eat meat presented in an advertisement. Indeed, that humans show empathy towards animals is commonly observed (Signal \& Taylor, 2007). 
Considering the suggested association between cuteness and empathy (Lishner et al., 2008), empathic responses might likely mediate the link between cuteness and meat consumption.

\section{Overview of the Present Studies}

In this article, we aimed to provide the first systematic investigation of the relationship between cuteness and meat consumption. Based on past literature, we hypothesized that empathy, humanization and caretaking behavior might play important roles in the association between cuteness and meat consumption. Across four pre-registered studies, including a total of 1074 participants, we tested these ideas using correlational and experimental designs.

In the first study, we aimed to replicate a model explaining meat consumption put forward by Kunst and Hohle (2016, Study 3). We also investigated the influence of cuteness perceptions as an additional, alternative mediator and its association with the other variables relating to meat consumption. The second study built on the model emerging from the first study, but manipulated the level of cuteness directly instead of investigating it as a mediator only. In the third study, we provided an extended and more methodologically sophisticated replication of the second study, including a wider variety of stimuli, caretaking intentions and general trait pro-social orientation measures to shed further light on the processes connecting cuteness responses with meat eating. Finally, we replicated the third study using a different population from a different country than the US to obtain information about the cross-cultural validity of our model (Study 3b).

Based on previous research we tested the following two main hypotheses across the four studies:

H1: Increased cuteness results in less willingness to consume meat

H2: The effect of cuteness on willingness to eat meat is mediated by empathy towards the animal 
We tested more specific hypotheses in some of the studies:

H3: The effect of cuteness on willingness to eat meat is mediated by humanization of the animal (Study 1)

H4: The effects of empathy on willingness to eat meat are mediated by a motivation of caretaking (Study 3a \& 3b)

H5: The effects of cuteness on willingness to eat meat and their mediated paths over empathy are moderated by general pro-social orientation (Study 3a \& 3b).

All four studies were pre-registered and analyses not included in the pre-registration are explicitly denoted as 'exploratory'. All additional analyses that fall within the main focus of the manuscript are included in the Supplementary Material. We report how we determined our sample sizes, all data exclusions (if any), all manipulations, and all measures in the studies (Simmons, Nelson, \& Simonsohn, 2012). Materials and data files are available at our project page (https://osf.io/rk4ut/) and all stimulus material and questionnaire items are available in the Supplementary Material. The studies were approved by the institutional review board of the University of Oslo, and all participants were provided with informed consent and were able to exit the study at any point in time.

\section{Study 1: Exploring Cuteness and Meat Consumption}

The rationale of the first study was twofold. First, we wanted to replicate the finding by Kunst and Hohle (2016) that presenting a (arguably cute-looking) lamb in a lamb chops advertisement results in reduced self-reported intention to consume the meat product. As in the original study, we expected these effects to be mediated by lowered dissociation of the meat from its animal origins and, subsequently, higher levels of empathy towards the animal. Different from the original study, however, we tested cuteness responses as an alternative first-stage mediator. That is, we tested whether showing the lamb in the advertisement would not only lead to more empathy toward the animal because of a lower degree of dissociation, 
but also because of heightened cuteness perceptions. Hence, the main focus of Study 1 was to explore the role of cuteness responses in light of the model proposed by Kunst and Hohle, which in turn could justify a more direct and confirmatory test of cuteness responses that we return to in Studies 2-3b.

\section{Method}

Participants. Sample size was identified with an a priori power calculation based on the effect reported by Kunst and Hohle (Cohen's $d=.487 ; \alpha=.05$, and $1-\beta=.95$ ). In total, we recruited $253^{1}$ US American participants (117 females, 1 other) on Amazon Mechanical Turk. We requested only US workers with at least $95 \%$ approval ratings and participants received $\$ 0.80$ as compensation. Participants' age ranged from 18 to 67 years $\left(M_{\text {age }}=35.16\right.$, $\left.S D_{\text {age }}=10.55\right)$. In total, $225(88.9 \%)$ participants reported eating meat and fish, while 16 $(6.3 \%)$ identified themselves as vegetarian or vegan. On average, participants reported consuming meat (including fish) on $M_{\text {meat }}=4.59$ days per week, and lamb specifically on $M_{\text {lamb }}=.43$ days per week.

Materials and procedure. The general procedure and materials were nearly identical to Study 3 by Kunst and Hohle (2016). However, based on our focus on cuteness we added some measures to the procedure. In the following section we present all measures:

After being presented with informed consent participants were first provided with a scale assessing trait dissociation adopted from Rothgerber (2013). As in the study by Kunst and Hohle (2016) the scale included three items $(\alpha=.91)$ such as "When I eat meat, I try not to think about the life of the animal I am eating", measured on a 7-point scale ranging from strongly disagree 1 to strongly agree 7. As Kunst und Hohle, we then presented four filler tasks in order to distract attention away from the purpose of the study.

\footnotetext{
${ }^{1}$ Two participants were excluded from the analyses. One had a large amount of missing or obviously fake responses (i.e., rating of 1 on nearly all items). The other indicated an age of 2 years.
} 
Next, participants were randomly assigned to one of two conditions. In the experimental condition, participants $(n=127)$ were presented with an advertisement for lamb chops including the picture of a lamb, while in the control condition, participants $(n=126)$ were shown the same advertisement without the animal present. These stimuli and conditions were identical to the ones employed by Kunst and Hohle (2016) and can be found in the supplementary material. For the next questions, the advertisement was always present at the top of the page, except for the demographics section at the end.

As in Study 3 by Kunst and Hohle (2016), participants completed two scales on empathy and state dissociation. The empathy scale presented five items $(\alpha=.95)$ created by Kunst and Hohle measuring empathy or empathic concern based on Davis (1980) and included items such as "Seeing the meat makes me feel pity for the animal that was slaughtered" rated on a 7-point scale ranging from strongly disagree 1 to strongly agree 7. The state dissociation scale $(\alpha=.79)$ included three items also taken from Kunst and Hohle (e.g., "The first thing I thought about when I saw the meat displayed above was a living being") and was measured on 7-point scales. These items were reverse-scored so that higher values meant more dissociation. Different to Kunst and Hohle, participants also completed a short scale assessing humanness $(\alpha=.97)$ in form of perceived similarity of the lamb to a human baby. Here, three items were rated (e.g., "The lamb intuitively reminds me of a human baby") on a 7-point scale anchored at not at all 0 and very much 6 . Finally, we employed a six-item scale $(\alpha=.87)$ assessing cuteness perceptions (Steinnes, 2017). The scale included items such as "The animal is cuddly" on a 5-point scale anchored at not at all 0 and very much 4.

The four scales were presented in random order and participants in both conditions were asked to complete the items with regard to the imagined animal that was the origin of the advertised meat. Having completed the scales, willingness to eat the meat presented in the 
advertisement was assessed by asking participants to indicate how negative or positive they felt about eating the meat on a slider ranging from extremely negative 0 to extremely positive 100. Finally, participants completed demographic information including questions on eating style (i.e., omnivore, pescetarian, vegetarian, or vegan) and how often per week they consumed meat in general and lamb specifically. Participants were then thanked and debriefed about the purposes of the study.

\section{Results}

Replication attempt of Kunst and Hohle (2016). Because of our focus on cuteness, we merely provide a brief overview of the findings. More detailed results are provided in the Supplementary Material. We were able to replicate the general main effect of the condition on willingness to eat the advertised meat. This willingness was higher in the control condition when the animal was not present. In addition, we also replicated the mediation model by Kunst and Hohle (2016) using path analysis. The effect of the experimental condition on willingness to eat meat was completely mediated by state dissociation and subsequent empathy (Supplementary Material Figure 1). However, we failed to find evidence for an interaction effect of trait dissociation and condition on either willingness to eat meat, empathy or state dissociation. That is, the experimental manipulation led to an increase of empathy, and a decrease of willingness to eat the meat and state dissociation ratings regardless of how participants scored on trait dissociation.

Cuteness and humanization. As pre-registered, we tested whether ratings of humanness and cuteness mediated the effect of our manipulation on willingness to eat the meat. A Welch's $t$-test indicated that cuteness responses towards the imagined lamb differed across the two conditions, with participants in the experimental condition giving higher 
cuteness ratings than those in the control condition did (Table 1$){ }^{2}$ Similarly, humanness ratings for the lamb also differed across the two conditions, with participants in the experimental condition giving higher humanness ratings than those in the control condition did (Table 1).

We fitted different models using path analysis in MPlus (Muthén \& Muthén, 2010). Indirect effects were tested using 10,000 bootstrap resamples. The first model tested whether humanization and, subsequently, cuteness mediated the effect of the experimental manipulation on willingness to eat meat in a two-stage mediation process. Here, we also explored whether humanness mediated the effects of the condition on cuteness as a first-stage mediator. In the fully-saturated model (see Figure 1), the lamb indirectly led to slightly lower levels of intentions to eat the meat, mediated by the first-stage mediator, humanness and the second-stage mediator, cuteness, $\beta=-.02$ [95\% Boot: -.04, -.01]. Moreover, showing the lamb indirectly lead to lower levels of willingness to eat meat mediated by heightened cuteness perceptions, $\beta=-.10$ [95\% Boot: -.15, -.05], as well as heightened humanness perceptions, $\beta=.06[95 \%$ Boot: $.02, .10]$. The path of condition on willingness to eat meat became insignificant when humanness and cuteness were added to the model, $\beta=-.11, B=-$ 7.14 [95\% Boot: $-15.15, .64]$, indicating full mediation.

Next, we extended the model of Kunst and Hohle (2016) in an exploratory fashion by adding the humanness-cuteness mediation previously observed (i.e., humanness being a firststage and cuteness a second-stage mediator). In this extended model, which showed poor model fit $\left(\mathrm{CFI}=.727, \mathrm{RMSEA}=.421, \chi^{2}(4)=183.04, p<.001\right)$, the indirect effect via the state dissociation-empathy mediation was significant, $\beta=-.21$ [95\% Boot: $-.28,-.15]$, while no indirect effect via the new humanness-cuteness mediation was observed, $\beta=.005[95 \%$

\footnotetext{
${ }^{2}$ In order to enhance the informational value of our statistical analyses (i.e. providing evidence for $\mathrm{H}_{0}$ ) we report the Bayes Factor calculated using JASP (JASP Team, 2017). All Bayes Factors were calculated using the default prior based on a Cauchy distribution $(0, .707)$. Similar to all significance tests, these are based on a twosided prior.
} 
Boot: $-.002, .01]$. Due to the poor model fit, we modified some paths based on modification indices. For instance, we regressed empathy on cuteness and also excluded humanness from the model because it explained the least variance in willingness to eat meat. Moreover, state dissociation and cuteness were allowed to correlate. Hence, this fitted model represented two paths, the Kunst and Hohle path over state dissociation and empathy and our new path over cuteness and empathy (see Figure 2). The model indicated good model fit (CFI = 1, RMSEA $=.02$ [90\% CI: $\left.0, .11], \chi^{2}(3)=3.43, p=.33\right)$. Showing the lamb indirectly increased empathy, mediated by heightened cuteness perceptions, $B=.73, \beta=.10[95 \%$ Boot: .05, .16], but especially mediated by lowered state dissociation, $B=.38, \beta=.20$ [95\% Boot: .13, .27], which was the significantly stronger indirect effect, $B_{\text {diff }}=.35[.01, .70]$.

In an exploratory fashion, we tested the cuteness-empathy link in a correlational model ignoring the experimental design and without the dissociation ratings. The cuteness ratings were employed as the predictor variable, empathy as the mediator and willingness to eat the meat as the outcome variable (Figure 3). The model was fully saturated and cuteness ratings did indirectly decrease the willingness to eat meat mediated by increased empathy, $\beta$ $=-.54[95 \%$ Boot: $-.62,-.45]$

\section{Discussion}

Study 1 presented a first correlational test of the role that cuteness may play for meat consumption. After successfully replicating the general model by Kunst and Hohle (2016), we evaluated a different path over humanness and cuteness. The path from the condition to willingness to eat meat was fully mediated by these variables. We then compared this mediation path to the one put forward by Kunst and Hohle. When included in the same model, the mediation path over state dissociation and empathy was much stronger and our new path had no effect on willingness to eat meat. However, in an exploratory fitted model, 
cuteness (together with state dissociation) mediated the effects of showing the lamb on empathy, suggesting that cuteness may be an antecedent of empathic responses.

Although humanness mediated the effect on cuteness, it contributed relatively little to explain variance in willingness to eat meat. We therefore turn to the stronger pathway in our models, which looks at cuteness and empathy, in the next studies. Cuteness has been experimentally linked to increased empathic reactions in previous research (Lishner et al., 2008). As our first study solely relied on the mediational function of cuteness, which in essence is correlational, a stronger test of its causal role would be to directly manipulate cuteness perceptions. In addition, Study 1 included a control condition without an animal present, which is not an optimal direct test. We provide such a more direct experimental test of cuteness perceptions in the next studies.

\section{Study 2: Experimentally Testing Whether Cuteness Influences Willingness to Eat Meat by Increasing Empathy}

The rationale of the second study was based on the final model explored in Study 1 (see Figure 3). By manipulating the cuteness of a presented animal, we wanted to first test its direct effect on willingness to eat meat and also whether this pathway would be mediated by empathy. We therefore employed similar materials and procedure as in Study 1 and dropped the dissociation and humanness items.

\section{Method}

Participants. We based our power calculations on the experimental effect of the cuteness manipulation on willingness to eat meat observed in Study $1(d=.49)$, but expected a smaller effect due to our different experimental manipulation that has not been tested before. Using $\alpha=.05$, and $1-\beta=.90$ yielded a total sample size of 382 for $d=.30$ using G*Power (Faul, Erdfelder, Lang, \& Buchner, 2007). Hence, a total of 407 US American participants were recruited on Amazon MTurk (199 females), with the age range going from 
18 to 75 years $\left(M_{\text {age }}=38.09, S D_{\text {age }}=12.29\right)$. We requested only US workers with at least $95 \%$ approval ratings and participants received $\$ 0.40$ as compensation. The sample included 386 participants identifying themselves as either omnivores or pescetarians (94.8\%) and 21 $(5.2 \%)$ as vegetarian or vegan. Participants reported on average consuming meat on $M=4.77$ $(S D=2.16)$ days per week and lamb specifically on $M=.47(S D=.82)$ days per week. In total, 201 participants were randomly assigned to the control and 206 to the experimental condition.

Materials and procedure. Participants were randomly allocated to a control condition (neutral lamb) or an experimental condition (cute lamb). Both conditions showed the version of the lamb chops advertisement from Study 1 that included the picture of the lamb. Crucially, however, using photo-editing software, the animal was either altered to look neutral or to look cute by changing aspects that typically are associated with a "baby-like" look, such as size of the eyes or the chin, round head shape or a high forehead (Kringelbach et al., 2016). As in Study 1, the advertisement was presented for all dependent variables. Please see the Supplementary Material for the stimuli used in each condition.

We utilized the same scales assessing cuteness $(\alpha=.90)$ and empathy $(\alpha=.97)$ as in Study 1 . These were presented in random order for each participant and the items were completed with regard to the advertisement. Next, participants indicated their hypothetical willingness to eat the advertised meat using the same slider scale as in Study 1 . We finally added an item assessing the angriness of the depicted animal (i.e., "The lamb looks angry") on a 5-point scale anchored at not at all 1 and very much 5, which constituted a control variable. We added this variable based on discussions with other scholars who pointed out that the control lamb might be perceived as angry-looking, which could be another factor influencing willingness to eat the dish. Participants then completed demographic information, 
an item targeting their preferred eating style and items asking about their frequency of meat consumption, and were finally debriefed.

\section{Results}

Supporting the effectiveness of our experimental manipulation, a Welch's $t$-test showed that participants characterized the lamb in the cute condition as cuter than the lamb in the neutral condition (Table 2).

Cuteness and willingness to eat meat. A Welch's $t$-test suggested that willingness to eat the lamb chops did not differ between the cuteness condition and the neutral control condition (Table 2). ${ }^{3}$ As pre-registered, we estimated a fully-saturated mediation model using path analysis in MPlus, testing whether perceived cuteness would mediate the effect of condition on willingness to eat meat (Supplementary Figure 2). Bootstrapping showed that this indirect effect was significant, $\beta=-.06$ [95\% Boot: -.10, -.03].

Empathy as a mediator. Based on the model in Study 1, we tested the second hypothesis that empathy would mediate the relation between the cuteness condition and willingness to eat the advertised meat. First, we checked whether empathy was differently reported for the two conditions using a Welch's $t$-test. On average, participants experienced more empathy towards the cute lamb than towards the neutral one, which was significant according to a traditional p-value criterion, while the Bayes Factor indicated no evidence for either $\mathrm{H}_{0}$ or $\mathrm{H}_{1}$ (Table 2). We then ran our fully saturated mediation model observing an indirect and negative effect of cuteness condition on willingness to eat meat as mediated by empathy, $\beta=-.10$ [95\% Boot: $-.18,-.01]$. The path between condition and willingness to eat meat was fully mediated by empathy (Figure 4). We repeated the mediation model in an exploratory fashion with the cuteness rating instead of the experimental manipulation as the predictor variable (Figure 3). In this fully-saturated model, we observed an indirect and

\footnotetext{
${ }^{3}$ The Bayes Factor indicates evidence for $\mathrm{H}_{0}$. In an exploratory fashion, we reanalyzed the difference while excluding the vegetarian and vegan participants. The difference between the condition was still non-significant.
} 
negative effect of cuteness rating on meat eating as mediated by empathy, $\beta=-.31[95 \%$ Boot: $-.40,-.23]$.

\section{Discussion}

Study 2 provided an experimental test of our main hypothesis $(\mathrm{H} 1)$ and tried to replicate our mediation model explored in Study 1 (H2). Contrary to H1, our data did not suggest a direct effect of manipulating cuteness on willingness to eat the advertised meat. Participants seeing the lamb that was photo-edited to be particularly cute, were on average not less willing to eat the lamb chops than participants seeing the neutral lamb. We also found some indication of a sex effect, with cuteness condition having a direct and positive effect on willingness to eat the meat among women. Surprisingly, this effect was in the opposite direction than expected (Supplementary Figure 4). We can only speculate that adding empathy as a mediator results in a suppressor effect, because the original association between the experimental condition and willingness to eat the meat was in the expected negative direction.

Providing some support for our predictions, we found a correlational effect via our cuteness variable on willingness to eat the meat. Specifically, participants reported higher cuteness scores in the cute condition, which, in turn, was associated with decreased intention to eat the advertised meat. Also, the mediation model proposed in Study 1 was replicated both experimentally and correlationally. Empathy fully mediated the path between the condition or the cuteness rating and willingness to eat the meat. This finding also held when controlling for angriness perceptions, but the experimental manipulation did not hold when controlling for sex.

A limitation of the present study is that it only focused on lamb meat and therefore only presented lamb stimuli. Participants indicated only rare consumption of lamb, which suggests a limited relevance of the study to their actual consumer habits. Consumption of 
pork and beef tend to be way more widespread and common among meat eaters in the US (OECD, 2016). Hence, extending our range of stimuli, we included these two animal types in the next study. In addition, empathy or empathic concern has repeatedly been shown to result in altruistic helping behavior (Batson, Fultz, \& Schoenrade, 1987; Batson et al., 2005).

Empathic reactions towards the animal might thereby result in increased intentions of helping or caretaking behavior, which might decrease people's willingness to eat meat. We, therefore, added a measure for caretaking as an additional mediator. Further, it has been proposed that responses to cuteness are evolutionarily grounded (Bradshaw \& Paul, 2010; Preston, 2013). Hence, our cuteness model might be influenced by the general disposition to care for young animals. We will also address this question in the next study. Finally, while we did not find an effect of angriness in Study 2, it is possible that participants are less willing to eat the cute animal because it is typically perceived as younger in age. Therefore, we also added a measure of perceived age in the next studies.

\section{Study 3a: Replicating and Extending Study 2}

The next two studies attempted to replicate and extend the major findings of Study 2 by addressing its limitations. Study 3 a was run using an US sample recruited as in the previous two studies. We aimed to replicate findings from Study 3a after analysis of its results by conducting the same study protocol in a different population, specifically, using Norwegian undergraduates (Study 3b). This would allow us to test the degree to which results are culture-dependent or can be generalized across two cultures in which the populations' exposure to farmed animals may differ. ${ }^{4}$ In contrast to Study 2, we included more varied stimuli in this study, including advertisements showing beef and pork, and animal stimuli showing calves and piglets in addition to lambs. We also added measures on caretaking

\footnotetext{
${ }^{4}$ Norway has twice as many farms per capita as the United States (Lowder, Skoet, \& Raney, 2016, Supplementary Material), and farmed animals spend more time outdoors in Norway. For instance, while fewer than $5 \%$ of the lactating cows in the United States have access to pasture during the grazing season (von Keyserlingk, Cestari, Franks, Fregonesi, \& Weary, 2017), all Norwegian cows are by law entitled to minimum eight weeks on pasture during the summer (Forskrift om hold av storfe, 2004).
} 
intentions and general trait pro-social orientation. We hypothesized that presenting cute animals in a meat advertisement results in reduced reported willingness to eat the advertised meat $(\mathrm{H} 1)$; the relation between the experimental manipulation and willingness to eat meat is mediated by empathy $(\mathrm{H} 2)$; the path between empathy and willingness to eat meat is further mediated by caretaking intentions (H4); the paths of this mediation model are moderated by general pro-social orientation (H5).

\section{Method}

Participants. The present study utilized a multilevel mixed design, which makes an a priori power analysis not as straightforward as in generalized linear methods. We, therefore, calculated our power based on a repeated measures ANOVA $(\alpha=.05,1-\beta=.80, f=.15)$ and oversampled the recommended sample size $(n=236)$. The final sample comprised of 306 (918 cases given the mixed design) US Americans (133 females) recruited on Amazon MTurk. We requested only US workers with at least 95\% approval ratings and participants received $\$ 1.11$ as compensation. Age ranged from 18 to 75 years $\left(M_{\mathrm{age}}=34.54, S D_{\mathrm{age}}=\right.$ 11.11). Of the sample, $96.4 \%$ identified themselves as meat eaters, while $3.6 \%$ reported a vegetarian or vegan eating style. Participants reported on average eating meat $M=4.59(S D=$ $2.08)$ times per week and lamb, beef and pork $M_{\text {lamb }}=.36(S D=.79), M_{\text {beef }}=2.27(S D=$ $1.48), M_{\text {pork }}=1.60(S D=1.34)$ times per week respectively.

Materials and procedure. After introducing the study, participants were randomly presented with two different scales measuring general pro-social orientation. The first measure on pro-social orientation was adapted from Sherman et al. (2013). The scale included six items adapted from the 30-item Moral Foundations Questionnaire assessing the care/harm value dimension. Here, participants indicated their agreement or disagreement with three statements (e.g., "Compassion for those who are suffering is the most crucial virtue") and the extent to which three different considerations are relevant to decide whether 
something is morally relevant or not (e.g., "Whether or not someone suffered emotionally"). Ratings were performed using a 6-point scale ranging from strongly disagree 1 to strongly agree 5 for the first three items and from not at all relevant 0 to extremely relevant 5 for the latter items. In addition, we included a second measure on pro-social orientation using Neel, Kenrick, White, and Neuberg's (2015) child care measure (e.g., "Providing for children is important to me"; disagree 1 - strongly agree 7). Having completed these potential moderators, participants completed the same filler tasks as in Study 1.

Next, participants were randomly presented with three of in total six versions of advertisements. Specifically, the study used a 3 (within-subject animal type factor: lamb, calf, pig) x 2 (between-subjects factor: cute vs. non-cute) mixed design (Judd, Westfall, \& Kenny, 2012). Hence, every participant was always presented with a lamb, a calf, and a piglet advertisement in random order. ${ }^{5}$ Importantly, for each advertisement, the animal displayed was either cute or neutral-looking. This between-factor was applied repeatedly to each trial, so that the number of advertisement showing cute-looking animals could range from 0 to 3 . The animal pictures used were successfully pre-tested in a pilot study, showing that they evoked the expected levels of cuteness responses ( $n=136$, see Supplementary Material). For each advertisement, participants completed the same empathy measure $(\alpha=.97)$ as in the previous studies. We added three items to measure caretaking intention $(\alpha=.92)$ of the animal on a 7-point scale anchored at strongly disagree 1 and strongly agree 7 . The scale included items such as "I want to protect animals such as the one used for producing the meat in the advertisement." The empathy and caretaking measures were always presented in random order. Afterwards, participants completed the same willingness to eat meat item as in the previous studies for each advertisement.

\footnotetext{
${ }^{5}$ We repeated all analyses, controlling for presentation effects. In general, we observed a few very small effects of order on cuteness and empathy, and no effects on willingness to eat the meat. In both Studies $3 \mathrm{a}$ and $3 \mathrm{~b}$, the pig was perceived as cuter in the non-cute condition when it was presented first in contrast to the second or third position. No effects were observed for the other animals.
} 
Finally, participants were shown all three advertisements again in random order and completed the same cuteness measure $(\alpha=.89)$ as in the previous studies. This was done at the end to prevent participants from inferring the purpose of the study and the cuteness manipulation in particular. We here also included the angriness item from Study 2 and as additional control variable added a new item assessing the perceived age of the animal on a 10-point scale anchored at very young 1 and very old 10.

After finishing these measures, participants completed demographic information and questions asking about their eating style and weekly consumption of meat as in the previous studies.

\section{Results}

Studies 3a and $3 \mathrm{~b}$ utilized a mixed approach and we analyzed our data using multilevel models in SPSS 24 in alignment with recent suggestions (Judd et al., 2012). For all models, intercepts, but not slopes were allowed to vary randomly according to participants. The estimation of mediation models is not straightforward using multilevel (e.g., in our study level 1: animal; level 2: cuteness) approaches. Some suggestions have been provided for clustered data (Bauer, Preacher, \& Gil, 2006; Preacher, Zyphur, \& Zhang, 2010), but our study contained crossed data. We therefore conducted mediation in a more traditional approach using multilevel models by first regressing the mediator $(\mathrm{m})$ on the predictor variable (x) to estimate path $a$, then regressing the outcome variable (y) on $\mathrm{m}$ and $\mathrm{x}$ in order to estimate paths $b$ and $c^{\prime}$. Path $c$ was estimated by regressing y on $\mathrm{x}$. Finally, we calculated a 95\% confidence interval around the indirect effect using a Monte Carlo procedure suitable for multilevel data developed by Falk and Biesanz (2016). For all analyses, we excluded participants identifying themselves as vegetarian or vegan, yielding a total of 295 participants. Results including these participants yielded similar results, though often slightly weaker effects. 
To test whether our manipulation induced different levels of cuteness, we regressed condition (cute vs. non-cute) and animal type (lamb vs. calf vs. pig), as well as their interaction on the cuteness score in a multilevel model. We observed a main effect for both condition, $F(1,692)=95.70, p<.001$, and animal type, $F(2,585)=90.10, p<.001$, but not for the interaction, $F(2,702)=.61, p=.545$. The cute condition evoked higher cuteness ratings than the non-cute condition (Table 3). Moreover, an inspection of the animal type effect showed that the lamb received higher cuteness ratings than the calf and the pig (Supplementary Table 3). We also tested for the effects of angriness and perceived age, which can be found in the Supplementary Material, but that played little of a role.

Cuteness and willingness to eat meat. In order to test the first hypothesis, we regressed the willingness to eat meat item on condition, animal type and their interaction. We observed both main effects for condition, $F(1,617)=18.32, p<.001$, and animal type, $F(2$, $585)=5.31, p=.005$, but no effect for the interactions, $F(2,620)=1.26, p=.285$. Willingness to eat meat was lowest for the lamb as compared to the calf and pig, which did not differ from each other. In addition, participants in the cute condition were on average less likely to consume the meat than people in the control condition were, supporting the first hypothesis (Table 3$)$.

Empathy as a mediator. Before running the mediation model, we regressed the empathy score on condition, animal type, and their interaction. Again, we observed both main effects for condition, $F(1,604)=14.53, p<.001$, and animal type, $F(2,585)=6.41, p=.002$, but no interaction. The lamb evoked higher empathy than the other animals. Moreover, participants reported higher empathy in the cuteness condition than in the control condition, as predicted (Table 3$)$.

Because we found a main effect of the cuteness condition on empathy, we fitted a mediation model using the condition as the independent variable (x), willingness to eat meat 
as the outcome variable (y) and empathy as the mediator (m). For reasons of analytic simplicity, we disregarded animal type when testing the paths. We first regressed empathy on condition to obtain path a, then willingness to eat meat on both condition and empathy to obtain paths b and c', and finally willingness to eat meat on condition to obtain path c. We found an indirect effect of the cuteness condition on meat eating that was mediated by empathy, $\beta=-.03, B=-2.14$ [95\% CI: $-3.39,-1.00]$. The direct effect of the cuteness manipulation on meat eating was still significant when adding empathy as a mediator, suggesting partial mediation (model overview Figure 4). Because the effect of the cuteness manipulation on empathy was low, we repeated the mediation model with the cuteness score as the independent variable. This time, empathy fully mediated the link between cuteness and willingness to eat, $\beta=-.11, B=-3.54$ [95\% CI: $-4.12,-2.93]$. High cuteness scores led to higher ratings of empathy, which in turn resulted in less reported willingness to eat the meat (full model overview Figure 3).

\section{Discussion}

Study $3 \mathrm{a}$ aimed to replicate the main findings of Study 2 and added two additional tests to the model. This time using a broader range of stimuli, we found a main effect of our cuteness manipulation on willingness to eat meat. This effect was more pronounced for men than for women (see Supplementary Material). We also replicated the mediation pathway from Study 2. Empathy ratings partially mediated the relationship between our experimental manipulation and willingness to eat meat. As in Study 2, this indirect effect was very small, mostly based on the small difference in empathy ratings between the conditions. Using the cuteness rating score in a correlational mediation model, we found a full mediation effect that was considerably stronger. The correlational effect was again more pronounced for females than for males. 
Extending the design of Study 2, we also tested the moderating effect of general prosocial orientation using two different measures as moderators in our mediation model. Both measures only moderated the path between empathy and willingness to eat meat, not the path between cuteness and empathy or cuteness and willingness to eat meat. Specifically, the measure assessing pro-social values (PSO-1) revealed that for individuals who scored high in contrast to low on this measure, empathy had a negative effect on willingness to eat meat (see Supplementary Material). Interestingly, this effect was reversed for individuals with low PSO-1 ratings. That is, for participants who scored low on pro-social values, more empathy meant a higher willingness to eat the meat. The second measure assessing the general tendency of childcare (PSO-2) showed only this reversed effect. Such a reverse effect is unexpected and might stem from the fact that only a small proportion scored high on the prosocial orientation measures and simultaneously provided low empathy ratings. Future studies would need to replicate this effect.

Finally, we tested whether caretaking intentions would help to further explain why empathy predicted a lower willingness to eat meat. Indeed, together, empathy and caretaking fully mediated the path between cuteness and willingness to eat meat (see Supplementary Material). Caretaking also partially mediated the path between empathy and willingness to eat meat. However, based on these findings, the unmediated effect of empathy on the willingness to eat meat was strongest.

We also tested whether our findings might be explained by the fact that our experimental manipulation induced differences in the perception of angriness and age of the animals. As in Study 2, we did not find evidence for such an alternative explanation. Empathy still provided an indirect effect, though only for the correlational mediation model when controlling for these alternative variables.

\section{Study 3b: Replicating Study 3a with a Different Population}


After analyzing Study 3a we conducted a replication study using Norwegian undergraduates. Although we did not have a specific rationale of why sampling Norwegian undergraduates we expected similar, though smaller effects due to the fact that a student sample might already be more critical towards meat consumption, and because exposure to farm animals is likely to be higher for the average consumer in Norway compared to the US (see Footnote 4). The materials and analyses followed the same pre-registration plan as Study 3a.

\section{Method}

In total, 117 Norwegian undergraduate students participated in the study for partial course credit. One participant was excluded because of missing data, and eight participants indicated their eating style as vegetarian or vegan, and were therefore excluded for the main analyses, as in Study 3a and as outlined in the pre-registration. The final sample consisted of 108 participants ( 84 females) ranging from 19 to 44 years of age $(M=21.53, S D=3.50)$. Participants reported on average eating meat $M=5.12(S D=1.88)$ times per week. ${ }^{6}$ We used the identical measures and stimuli as in Study 3a. Items were translated into Norwegian. The translation was then discussed between the authors and the translator, who were bilinguals. However, we did not employ a back-translation method (Brislin, 1970), which is a limitation of the current study.

\section{Results}

To test whether our manipulation induced different levels of cuteness, we regressed condition (cute vs. non-cute) and animal type (lamb vs. calf vs. pig), as well as their interaction on the cuteness score in a multilevel model. We observed a main effect both for condition, $F(1,244)=47.65, p<.001$, and animal type, $F(2,211)=24.95, p<.001$, but not for the interaction, $F(2,243)=1.71, p=.184$. As in Study 3a, the cute condition evoked

\footnotetext{
${ }^{6}$ Items on average weekly consumption of beef, lamb and pork were administered, but included a clerical error so that each item asked about weekly consumption of chicken. As this invalidates these responses, they are not presented here.
} 
higher cuteness ratings than the non-cute condition. Moreover, an inspection of the animal type effect showed that the lamb received higher cuteness ratings than the calf and the pig (Supplementary Table 4). We also tested for the effects of angriness and perceived age, which can be found in the Supplementary Material, but found again only minor effects.

Cuteness and Willingness to Eat Meat. In order to test the first hypothesis, we regressed the willingness to eat meat item on condition, animal type and their interaction. We observed only a main effect of animal type, $F(1,211)=6.46, p=.002$, but no effect of condition, $F(2,223)=1.42, p=.234$, nor of the interaction, $F(2,223)=.87, p=.422$. In contrast to Study 3a, willingness to eat meat was lowest for the pig, but did not differ between the lamb and calf. In addition, as opposed to findings in Study 3a, participants in the cute condition were on average not less likely to consume the meat than people in the control condition were (Table 4).

Empathy as a Mediator. Before running the mediation model, we regressed the empathy score on condition, animal type and their interaction. We observed a main effect of condition, $F(1,220)=20.26, p<.001$, but not of animal type, $F(2,211)=1.04, p=.356$, or the interaction, $F(2,219)=.13, p=.877$. Participants reported higher empathy in the cuteness condition than in the control condition (Table 4).

Because we found a main effect of condition on empathy, we fitted a mediation model using the condition as independent variable (x), willingness to eat as the outcome variable (y) and empathy as the mediator (m). For reasons of analytic simplicity, we disregarded animal type when testing the paths. As in Study 3a, we first regressed empathy on condition to obtain path a, then willingness to eat on both condition and empathy to obtain paths b and c', and finally willingness to eat on condition to obtain path c. We replicated an indirect effect of the cuteness condition on meat eating that was mediated by empathy, $\beta=-.04, B=-2.46[-$ $4.16,-1.14]$. The direct effect of the manipulation on the dependent variable was not 
significant when adding the mediator, suggesting full mediation (see full model overview in Figure 4). As in Study 3a, we repeated the mediation model with the cuteness score as independent variable. This time, empathy partially mediated the link between cuteness and willingness to eat meat, $\beta=-.07, B=-2.27[-3.08,-1.43]$. High cuteness scores led to higher ratings of empathy, which in turn resulted in less reported willingness to eat the meat (see full model overview in Figure 3). The cuteness rating in this model exhibited a positive effect on willingness to eat, contradictory to our expectations and to the findings from Study $3 a$.

\section{Discussion}

Study $3 \mathrm{~b}$ aimed to replicate Study $3 \mathrm{a}$ using a different population. In sum, we replicated the mediation effect of empathy on willingness to eat meat using both the cuteness manipulation and the cuteness perception ratings. In contrast, to study $3 \mathrm{a}$ we did not find a direct effect of our cuteness manipulation on willingness to eat meat. In addition, we did not replicate the findings of caretaking intentions and the moderating role of pro-social intentions (Supplementary Material). We will return to a detailed discussion of these findings and their successful or non-successful replications in the General Discussion.

\section{General Discussion}

In this article, we explored the effect of cuteness perceptions on the intention to eat meat. Across four studies, we tested different hypotheses. In Study 1, we conducted a preliminary test of the role that cuteness may play by extending a model of Kunst and Hohle (2016). While cuteness seemed to play less of a direct role for meat consumption than empathy towards the slaughtered animal did, our models suggested that cuteness perceptions may precede such empathic responses and thereby lead to lowered willingness to consume meat. In Studies 2, 3a, and 3b, we then directly manipulated cuteness by altering facial features of animals used for food consumption. Evidence was mixed, but to some extent supported the view that increased cuteness results in less willingness to consume meat in the 
US, but to a lesser extent in Norway. Importantly, results from all studies were consistent in that the effect of cuteness on willingness to eat meat was mediated by empathy towards the animal.

\section{Cuteness and Meat Consumption}

Overall, we obtained correlational and experimental evidence that the cuter consumers perceived animals to be, the less inclined they were to eat the animal's meat, which is consistent with findings from a recent correlational study (Ruby \& Heine, 2012). Indeed, speaking to the robustness of the observed effects, meta-analyzing all five studies (including the pilot study, see Supplementary Material), we observed an overall effect size of $r=-.21[-.38,-.04]$ (see Figure 5), demonstrating that higher ratings of cuteness for different animals coincided with lower willingness to eat an advertised meat dish ostensibly related to the animal. Interestingly, this effect was only present in the U.S samples, but not in the Norwegian sample. Effects for females tended to be stronger, though this sex difference was not significant, $z=.36, p=.72$. Meta-analyzing the experimental results from Studies 2 and $3 \mathrm{a} / \mathrm{b}$ and the pilot study revealed an overall effect size of $r=-.05[-.09,-.004]$, for the experimental manipulation on willingness to eat the meat. ${ }^{7}$ Although we did not observe a significant direct effect in the pilot study, Study 2, and Study 3b, the overall difference across all studies was significant, though not substantial in size (Figure 6). This effect was not moderated by sex in the meta-analysis, $z=.54, p=.59$. Yet, considering the small effect size, the practical significance of the effect can be questioned. On the one hand, it seems that our cuteness manipulation, although inducing on average different levels of cuteness responses in each study, could have been stronger. To increase experimental control, we chose to use the same young animal in both conditions and only altered its cuteness visually. This may however have led to relatively modest cuteness differences. In fact, most animals in the

\footnotetext{
7 This transforms into a Cohen's $d$ effect size of .10, which according to typical suggestions (Cohen, 1988) refers to no effect, while others have labeled it a developmental effect (Hattie, 2009).
} 
control condition were already perceived as cuter than the midpoint of our measure. Future studies could therefore manipulate cuteness using less cute animals as control stimuli at the expense of experimental control, and while controlling for perceived age, anger, or other confounding variables. Alternatively, it is also possible that cuteness does not exert a strong influence on meat consumption directly, but indirectly through other variables such as empathy. Our findings point in such a direction.

\section{The Role of Empathy}

In Studies 2 and 3a/b, empathy mediated the link between our experimental cuteness manipulations and willingness to eat the advertised meat. The overall indirect effect size across the three Studies $(2,3 \mathrm{a}, 3 \mathrm{~b})$ was $b=-.04$ [95\% CI: -.07, -.01], using a meta-analytic structural equation modeling approach (MASEM) as outlined by Cheung and Cheung (2016). This indirect effect was very small, due to the fact that empathy ratings did not differ strongly between conditions. Using a correlational mediation model in which we replaced the cuteness manipulation with the continuous cuteness measure, this indirect effect was much more pronounced $(b=-.25$, [95\% CI: -.42, -.07], meta-analyzing all four studies).

Our findings of the importance of empathy are not surprising. As demonstrated by Lishner et al. (2008), cute pictures of humans often result in more empathy, which was replicated with animal stimuli in the present studies. Kunst and Hohle (2016) also identified empathy as a potent mediator of effects on willingness to eat meat. In their model, not relating meat to its animal origins resulted in less empathy and thereby higher willingness to consume the meat. We provide evidence for an additional pathway, where perceiving an animal as cute results in increased empathy and thereby reduced willingness to eat meat, which may suggest the use of a modified version of the model by Kunst and Hohle in contexts were cuteness perceptions are relevant (e.g., when stimuli are visible and potentially cute). Cuteness may make the dissociation process more difficult. If cuteness has an 
evolutionary function inducing protection responses, it may be more difficult to ignore the fact that the meat on the plate is an animal, if its animal source is known to be cute. Likewise, when dissociation is made difficult (e.g., by showing the picture of the animal), cuter animals may decrease willingness to eat meat more than non-cute animals, while cuteness is less important when we are not reminded of the animal origin. The relationship between dissociation and cuteness may be further tested in future research.

\section{The Role of Caretaking}

In Studies 3a and 3b, we also included a measure of caretaking, which negatively predicted willingness to eat meat in Study 3a and was positively associated with empathy ratings. Although we hypothesized that caretaking would mediate the path from empathy to willingness to eat meat, it did so only partially in Study 3a and not at all in Study 3b. In both studies, empathy had a considerable influence on willingness to eat meat. Of course, because this relationship was correlational it cannot provide any causal inferences regarding the role of caretaking. We still observed similar effects as reported in past literature investigating caretaking behavior targeted at other humans (Nittono et al., 2012; Sherman et al., 2009, 2013), but only in the US sample. Specifically, in Study 3a, caretaking was positively associated with cuteness responses and also with empathy. These effects were not replicated with Norwegian undergraduates. Although cuteness ratings also correlated positively with ratings on caretaking, these ratings, in turn, did not correlate with Norwegian participants' willingness to eat the meat. This finding might be explained by cultural differences in interpretation of the caretaking measure. Caretaking tendencies did also not differ for the two conditions, providing further support that our experimental manipulation may have been too subtle.

Previous literature has also linked cuteness to the process of humanization (Sherman \& Haidt, 2011). We included humanization in Study 1, and found that it was positively 
related to cuteness perceptions. However, as empathy was a much stronger indicator of willingness to consume meat, we dropped humanization for the follow-up studies. This potentially limited role of humanization processes for meat consumption in consumer choice situations is consistent with previous research (e.g., Kunst and Hohle, 2016).

\section{The Evolutionary Importance of Cuteness}

Some authors have argued that cuteness perceptions trigger increased empathy or caretaking behavior based on an evolutionary mechanism to enhance the survival fitness of the human target (Leitão \& Castelo-Branco, 2010; Preston, 2013). In Study 3a and 3b, we included two measures targeted at inter-individual differences of pro-social orientation, to test whether such a mechanism could also be at play with animal targets. One measure assessed more general endorsement of pro-social values (Sherman et al., 2013), while the other measure was tapping child caring attitudes (Neel et al., 2015). In Study 3a, these measures only had a moderating effect on the path between empathy and willingness to eat meat, with the child caring measure only exhibiting differences for low empathy ratings. For the pro-social value measure, participants scoring high on the measure reported less willingness to eat the meat the more empathy they felt, but those with low pro-social values were indeed more willing to eat meat the more empathy they felt. However, these findings were not replicated in Study $3 \mathrm{~b}$ where pro-social values did not moderate the path between empathy and willingness to eat meat. The findings in Study 3a are therefore only tentative and need to be followed up in future research.

From an evolutionary perspective, one might raise the question why one should want to enhance the fitness of farm animals that have the typical function of meat production for humans. One possibility is that animals are just "social parasites" who release and exploit humans' evolved nurture instincts for baby schema features (Archer, 1997; Lorenz, 1943). Because human babies are so vulnerable and needy of help, the human cuteness detector may 
be set at such a low bar that anything remotely resembling a human baby is considered cute; including other mammal infants, or even non-living objects like cars (Miesler, Leder, \& Herrmann, 2011). Moreover, the cuteness effect is most pronounced for domestic animals or pets, which are often perceived as similar to humans (Kwan, Gosling, \& John, 2008). Such effects may have diffused to other animals, though in a less pronounced manner. One may also speculate that a cuteness response for farm animals serves an adaptive purpose for humans. Because humans have always relied on animal products for their survival, living animals have been a valuable resource. Ever since animals were domesticated some thousands of years ago, the relationship between humans and animals has been a nonegalitarian one, where humans have power over the animals, and animals depend on their human owner for survival (Serpell, 1996). Protecting especially the young and vulnerable domesticated animals from dangers until they reached slaughter age may therefore have been adaptive. Future research could test this proposition, comparing cuteness responses toward domesticated and non-domesticated animals.

Past research has identified sex differences with regard to cuteness (Lobmaier et al., $2015,2010)$. In the present studies, we also observed that females were more likely to score higher on our main variables such as cuteness, empathy, or caretaking, while simultaneously providing lower scores on the willingness to eat the advertised meat. In one study, the indirect negative effect through empathy was also significantly stronger for females than males. In general, these findings may be seen as providing further evidence for a possible evolutionary and biological basis of cuteness perceptions.

\section{Limitations}

Across the studies, we presented three different animals, namely a lamb, a calf and a piglet. While this variation may increase the generalizability of our results for mammals, it remains uncertain whether they apply to poultry or fish. It would be interesting in future 
research to explore the boundaries of the cuteness effect and to test whether it holds for nonmammal animal types which might be generally regarded as less cute. We would also like to highlight that the cuteness effect might be cultural specific and not extend to situations where consumption of specific meat is forbidden regardless of the animal's perception. It is for example questionable whether the piglet would have produced similar cuteness effects on the willingness to eat meat in cultures where it is morally condemned to eat pork (e.g., Judaism, Islam).

Results from Study $3 b$ indicated no direct effect of the experimental manipulation or the cuteness ratings on willingness to eat the meat. When adding empathy as a mediator, cuteness ratings actually indicated a positive effect on willingness to eat the meat. Whether this is a stable effect that is limited to the Norwegian or Scandinavian culture or just an artifact would need to be investigated in future studies. Comparing descriptives of Study $3 \mathrm{a}$ and $3 \mathrm{~b}$ reveals that the willingness to eat meat was higher in the Norwegian sample compared to the American sample. A tentative explanation might be that Norwegians are more used to seeing the animals they eat and therefore dissociate meat less from the animal in their daily lives, but still manage to eat it because they are used to the thought. In turn, this could make them less sensitive to the cuteness of animals as well. Another explanation might be that Study 3 b included a considerably lower number of subjects than Study 3a. Although employing a powerful mixed within-between subjects design, Study $3 b$ might not have been adequately powered to detect an effect.

Similarly, a paradox involves that, although young animals generally evoke more cuteness responses than older animals (Borgi et al., 2014; Little, 2012), many consumers prefer meat from young animals. Consumers prefer juicy and tender meat (Aaslyng et al., 2007; Huffman et al., 1996; Norman, Berg, Heymann, \& Lorenzen, 2003), and judge meat from young lambs as more tender and juicy than meat from older lambs (Sañudo et al., 2007), 
and suckling pig as more tender than meat from older pigs (Aaslyng et al., 2007). Studies also indicate that consumers are willing to pay more for meat from young animals (Jabbar, Baker, \& Fadiga, 2010). At the same time, because animals are bred and fed more efficiently than before, animals are also slaughtered at a younger age than before (Cochran, 2011). The U.S. Department of Agriculture recently revised its poultry definitions, lowering the ages of poultry classes such as fryer chicken and roaster, to "reflect the increasingly short time needed to bring U.S. poultry to market" (Reuters, 2011, p. 1). For instance, while a roaster was previously defined as a chicken aged three to five months, its slaughter age is now between eight and twelve weeks (Cochran, 2011). Moreover, meat from very young animals is often considered a delicacy, including veal cutlets, suckling pigs, spring lambs and Cornish Game hens (Ozersky, 2011). In light of this, one could also hypothesize that cuteness would increase willingness to eat an animal, because it indicates youth and tender meat. Such a process may be more likely in consumer-choice situations where dissociation is high and not interrupted by presentation of animal stimuli such as in our studies.

We would also draw awareness to the fact that the present research tested relatively short-lived and immediate cuteness responses. Cuteness effects on meat consumption might operate differently with regard to long-term effects. On the one hand, our studies suggest that cuteness effects might operate via empathy, which could have important implications for short-term effects of advertisements or campaigns targeting meat consumption. It is possible that repeated exposure to cuteness eliciting stimuli over a longer period of time might be necessary to produce long-lasting effects on willingness to consume meat. Future research could test this proposition and explore whether cuteness-eliciting stimuli in advertisements or campgains represent a potent factor of reducing meat consumption in the long run.

Last, the present studies used a straight-forward online procedure, which allowed us to control a number of extraneous variables, but resulted in low ecological validity. For 
instance, we asked participants about their hypothetical intentions to consume the advertised meat. Needless to say, follow-up studies should manipulate cuteness effects in laboratory or, optimally, in real life settings that allow for the assessment of actual meat consumption and choice. In addition, most participants in the present studies were recruited using Amazon MTurk. Although MTurk participants have been evaluated as comparable, or even superior to typical undergraduates, they often reveal attention biases and fatigue (Goodman, Cryder, \& Cheema, 2013; Peer, Brandimarte, Samat, \& Acquisti, 2017). We also did not include attention or accuracy checks in our present studies because completion time was typically minimal (sometimes below two minutes). This could have been done to ensure data quality. Yet, manipulation checks that we included supported the general effectiveness of our manipulation, possibly rendering the issue of including attention checks less important.

\section{Conclusion}

The current paper presented the first comprehensive empirical test of the effects of cuteness perceptions on people's willingness to consume meat. Cuteness perceptions indeed had negative correlational associations with willingness to consume meat and we also obtained some weak evidence of it having a causal effect on meat consumption. Moreover, testing and comparing different models suggested that the link between cuteness and willingness to eat meat is mediated by empathy towards the slaughtered animals. In other words, the cuter consumers perceive animals to be, the more empathy they feel towards them, making them less inclined to eat the meat. Hence, the present studies suggest that when animals are cute enough, they might become too sweet to eat. 


\section{Conflict of Interest}

The authors declare that the research was conducted in the absence of any commercial or financial relationships that could be construed as a potential conflict of interest.

\section{Author Contributions}

JZ and JRK conceived and designed the studies. JZ conducted the studies and the statistical analyses. JZ wrote the first draft, and all authors revised the final manuscript.

\section{Funding}

This research was supported by funding from the University of Oslo.

\section{Acknowledgements}

We wish to thank Kamilla K. Steinnes for providing us with the cuteness scale. 


\section{References}

Aaslyng, M. D., Oksama, M., Olsen, E. V., Bejerholm, C., Baltzer, M., Andersen, G., ... Gabrielsen, G. (2007). The impact of sensory quality of pork on consumer preference. Meat Science, 76(1), 61-73.

Adams, C. J. (2015). The sexual politics of meat: A feminist-vegetarian critical theory. New York, NY: Continuum.

Aragón, O. R., Clark, M. S., Dyer, R. L., \& Bargh, J. A. (2015). Dimorphous Expressions of Positive Emotion Displays of Both Care and Aggression in Response to Cute Stimuli. Psychological Science, 956797614561044.

Archer, J. (1997). Why do people love their pets? Evolution and Human Behavior, 18(4), 237-259.

Batson, C. D., Fultz, J., \& Schoenrade, P. A. (1987). Distress and Empathy: Two Qualitatively Distinct Vicarious Emotions with Different Motivational Consequences. Journal of Personality, 55(1), 19-39. https://doi.org/10.1111/j.1467-6494.1987.tb00426.x

Batson, C. D., Lishner, D. A., Cook, J., \& Sawyer, S. (2005). Similarity and Nurturance: Two Possible Sources of Empathy for Strangers. Basic and Applied Social Psychology, 27(1), 15-25. https://doi.org/10.1207/s15324834basp2701_2

Bauer, D. J., Preacher, K. J., \& Gil, K. M. (2006). Conceptualizing and testing random indirect effects and moderated mediation in multilevel models: New procedures and recommendations. Psychological Methods, 11(2), 142-163. https://doi.org/10.1037/1082-989X.11.2.142

Bohm, I., Lindblom, C., Åbacka, G., Bengs, C., \& Hörnell, A. (2015). “He just has to like ham"-The centrality of meat in home and consumer studies. Appetite, 95, 101112. 
Borgi, M., Cogliati-Dezza, I., Brelsford, V., Meints, K., \& Cirulli, F. (2014). Baby schema in human and animal faces induces cuteness perception and gaze allocation in children. Frontiers in Psychology, 5. Retrieved from http://www.ncbi.nlm.nih.gov/pmc/articles/PMC4019884/

Bradshaw, J., \& Paul, E. (2010). Could empathy for animals have been an adaptation in the evolution of Homo sapiens? Animal Welfare, 19(2), 107-112.

Bratanova, B., Loughnan, S., \& Bastian, B. (2011). The effect of categorization as food on the perceived moral standing of animals. Appetite, 57(1), 193-196. https://doi.org/10.1016/j.appet.2011.04.020

Brislin, R. W. (1970). Back-translation for cross-cultural research. Journal of CrossCultural Psychology, 1(3), 185-216.

Buckley, R. C. (2016). Aww: The Emotion of Perceiving Cuteness. Theoretical and Philosophical Psychology, 1740. https://doi.org/10.3389/fpsyg.2016.01740

Cerjak, M., Karolyi, D., \& Mesić, Ž. (2011). Consumers' attitudes towards farm animal welfare and their influence on meat consumption. Agriculturae Conspectus Scientificus (ACS), 76(4), 283-286.

Cheung, M. W.-L., \& Cheung, S. F. (2016). Random-effects models for meta-analytic structural equation modeling: review, issues, and illustrations. Research Synthesis Methods, 7(2), 140-155.

Cochran, C. (2011). Poultry Classifications Get a 21st Century Upgrade. Retrieved from https://www.usda.gov/media/blog/2011/11/17/poultry-classifications-get21st-century-upgrade

Cohen, J. (1988). Statistical power analysis for the behavioral sciences (2nd ed.). Hillsdale, NJ: Erlbaum. 
Davis, M. H. (1980). A multidimensional approach to individual differences in empathy. JSAS Catalog of Selected Documents in Psychology, 10, 85.

Falk, C. F., \& Biesanz, J. C. (2016). Two cross-platform programs for inferences and interval estimation about indirect effects in mediational models. SAGE Open, 6(1), 2158244015625445.

Faul, F., Erdfelder, E., Lang, A.-G., \& Buchner, A. (2007). G* Power 3: A flexible statistical power analysis program for the social, behavioral, and biomedical sciences. Behavior Research Methods, 39(2), 175-191.

Forskrift om hold av storfe. Forskrift om hold av storfe [Regulation about keeping cattle], Pub. L. No. 665 (2004). Retrieved from https://lovdata.no/dokument/SF/forskrift/2004-04-22-665

Glocker, M. L., Langleben, D. D., Ruparel, K., Loughead, J. W., Gur, R. C., \& Sachser, N. (2009). Baby Schema in Infant Faces Induces Cuteness Perception and Motivation for Caretaking in Adults. Ethology, 115(3), 257-263. https://doi.org/10.1111/j.1439-0310.2008.01603.x

Goodman, J. K., Cryder, C. E., \& Cheema, A. (2013). Data Collection in a Flat World: The Strengths and Weaknesses of Mechanical Turk Samples. Journal of Behavioral Decision Making, 26(3), 213-224. https://doi.org/10.1002/bdm.1753

Grauerholz, L. (2007). Cute Enough to Eat: The Transformation of Animals into Meat for Human Consumption in Commercialized Images. Humanity \& Society, 31(4), 334354. https://doi.org/10.1177/016059760703100404

Hattie, J. (2009). Visible Learning. London, UK: Routledge.

Huffman, K. L., Miller, M. F., Hoover, L. C., Wu, C. K., Brittin, H. C., \& Ramsey, C. B. (1996). Effect of beef tenderness on consumer satisfaction with steaks consumed in the home and restaurant. Journal of Animal Science, 74(1), 91-97. 
Jabbar, M. A., Baker, D., \& Fadiga, M. L. (2010). Demand for livestock products in developing countries with a focus on quality and safety attributes: evidence from Asia and Africa. Addis Ababa, Ethiopia: International Livestock Research Institute.

JASP Team. (2017). JASP (Version 0.8.1.1) [Computer software].

Judd, C. M., Westfall, J., \& Kenny, D. A. (2012). Treating stimuli as a random factor in social psychology: A new and comprehensive solution to a pervasive but largely ignored problem. Journal of Personality and Social Psychology, 103(1), 54-69. https://doi.org/10.1037/a0028347

Keating, C. F., Randall, D. W., Kendrick, T., \& Gutshall, K. A. (2003). Do babyfaced adults receive more help? The (cross-cultural) case of the lost resume. Journal of Nonverbal Behavior, 27(2), 89-109.

Kringelbach, M. L., Stark, E. A., Alexander, C., Bornstein, M. H., \& Stein, A. (2016). On Cuteness: Unlocking the Parental Brain and Beyond. Trends in Cognitive Sciences. Retrieved from http://www.sciencedirect.com/science/article/pii/S1364661316300420

Kunst, J. R., \& Hohle, S. M. (2016). Meat eaters by dissociation: How we present, prepare and talk about meat increases willingness to eat meat by reducing empathy and disgust. Appetite, 105, 758-774. https://doi.org/10.1016/j.appet.2016.07.009

Kwan, V. S. Y., Gosling, S. D., \& John, O. P. (2008). Anthropomorphism as a Special Case of Social Perception: A Cross-Species Social Relations Model Analysis of Humans and Dogs. Social Cognition, 26(2), 129-142. https://doi.org/10.1521/soco.2008.26.2.129

Leitão, M., \& Castelo-Branco, R. (2010). Babies: the irresistible power of cuteness. A study concerning the evolutionary function of infantile traits. Estudos de 
Psicologia (Natal), 15(1), 71-78. https://doi.org/10.1590/S1413$294 X 2010000100010$

Lishner, D. A., Oceja, L. V., Stocks, E. L., \& Zaspel, K. (2008). The effect of infant-like characteristics on empathic concern for adults in need. Motivation and Emotion, $32(4), 270-277$.

Little, A. C. (2012). Manipulation of infant-like traits affects perceived cuteness of infant, adult and cat faces. Ethology, 118(8), 775-782.

Lobmaier, J. S., Probst, F., Perrett, D. I., \& Heinrichs, M. (2015). Menstrual cycle phase affects discrimination of infant cuteness. Hormones and Behavior, 70, 1-6. https://doi.org/10.1016/j.yhbeh.2015.02.001

Lobmaier, J. S., Sprengelmeyer, R., Wiffen, B., \& Perrett, D. I. (2010). Female and male responses to cuteness, age and emotion in infant faces. Evolution and Human Behavior, 31(1), 16-21. https://doi.org/10.1016/j.evolhumbehav.2009.05.004

Lorenz, K. (1943). Die angeborenen formen möglicher erfahrung. Zeitschrift Für Tierpsychologie, 5(2), 235-409.

Loughnan, S., Haslam, N., \& Bastian, B. (2010). The role of meat consumption in the denial of moral status and mind to meat animals. Appetite, 55(1), 156-159. https://doi.org/10.1016/j.appet.2010.05.043

Lowder, S. K., Skoet, J., \& Raney, T. (2016). The number, size, and distribution of farms, smallholder farms, and family farms worldwide. World Development, 87, 16-29.

Luo, L., Kendrick, K. M., Li, H., \& Lee, K. (2015). Adults with siblings like children's faces more than those without. Journal of Experimental Child Psychology, 129, 148-156. https://doi.org/10.1016/j.jecp.2014.09.003

Miesler, L., Leder, H., \& Herrmann, A. (2011). Isn't it cute: An evolutionary perspective of baby-schema effects in visual product designs. International Journal of Design, 
5(3). Retrieved from

http://search.proquest.com/openview/69a1a08c6ffe965ebcc7b7b38fbf3a45/1? pq-origsite $=$ gscholar $\& \mathrm{cbl}=466416$

Muthén, L. K., \& Muthén, B. O. (2010). Mplus User's Guide: Statistical Analysis with Latent Variables: Users Guide (Vol. Version 6). Los Angeles, CA: Muthén \& Muthén.

Neel, R., Kenrick, D. T., White, A. E., \& Neuberg, S. L. (2015). Individual differences in fundamental social motives. Retrieved from http://psycnet.apa.org/psycinfo/2015-42189-001/

Nittono, H., Fukushima, M., Yano, A., \& Moriya, H. (2012). The Power of Kawaii : Viewing Cute Images Promotes a Careful Behavior and Narrows Attentional Focus. PLOS ONE, 7(9), e46362. https://doi.org/10.1371/journal.pone.0046362

Norman, J. L., Berg, E. P., Heymann, H., \& Lorenzen, C. L. (2003). Pork loin color relative to sensory and instrumental tenderness and consumer acceptance. Meat Science, 65(2), 927-933.

OECD. (2016). OECD-FAO Agricultural Outlook (No. 1563-447). Paris: Organisation for Economic Co-operation and Development. Retrieved from http://www.oecdilibrary.org/content/serial/19991142

Ozersky, J. (2011, October). The Gastronomic Case Against Eating Baby Animals. Time. Retrieved from http://ideas.time.com/2011/10/05/the-gastronomic-caseagainst-eating-baby-animals/

Parsons, C. E., Young, K. S., Kumari, N., Stein, A., \& Kringelbach, M. L. (2011). The Motivational Salience of Infant Faces Is Similar for Men and Women. PLOS ONE, 6(5), e20632. https://doi.org/10.1371/journal.pone.0020632 
Peer, E., Brandimarte, L., Samat, S., \& Acquisti, A. (2017). Beyond the Turk: Alternative platforms for crowdsourcing behavioral research. Journal of Experimental Social Psychology, 70, 153-163. https://doi.org/10.1016/j.jesp.2017.01.006

Piazza, J., Ruby, M. B., Loughnan, S., Luong, M., Kulik, J., Watkins, H. M., \& Seigerman, M. (2015). Rationalizing meat consumption. The 4Ns. Appetite, 91, 114-128.

Preacher, K. J., Zyphur, M. J., \& Zhang, Z. (2010). A general multilevel SEM framework for assessing multilevel mediation. Psychological Methods, 15(3), 209.

Preston, S. D. (2013). The origins of altruism in offspring care. Psychological Bulletin, $139(6), 1305$.

Reuters. (2011, November 2). Fast-growing fowl bring US poultry class revisions. Reuters. Retrieved from http://www.reuters.com/article/usa-poultryidUSN1E7A10LV20111102

Rothgerber, H. (2013a). A meaty matter. Pet diet and the vegetarian's dilemma. Appetite, $68,76-82$.

Rothgerber, H. (2013b). Real men don't eat (vegetable) quiche: Masculinity and the justification of meat consumption. Psychology of Men \& Masculinity, 14(4), 363.

Rothgerber, H., \& Mican, F. (2014). Childhood pet ownership, attachment to pets, and subsequent meat avoidance. The mediating role of empathy toward animals. Appetite, 79, 11-17.

Ruby, M. B., \& Heine, S. J. (2012). Too close to home. Factors predicting meat avoidance. Appetite, 59(1), 47-52. https://doi.org/10.1016/j.appet.2012.03.020

Sañudo, C., Alfonso, M., San Julián, R., Thorkelsson, G., Valdimarsdottir, T., Zygoyiannis, D., ... others. (2007). Regional variation in the hedonic evaluation of lamb meat from diverse production systems by consumers in six European countries. Meat Science, 75(4), 610-621. 
Serpell, J. (1996). In the company of animals: A study of human-animal relationships. Cambridge, UK: Cambridge University Press.

Sherman, G. D., \& Haidt, J. (2011). Cuteness and Disgust: The Humanizing and Dehumanizing Effects of Emotion. Emotion Review, 3(3), 245-251. https://doi.org/10.1177/1754073911402396

Sherman, G. D., Haidt, J., \& Coan, J. A. (2009). Viewing cute images increases behavioral carefulness. Emotion, 9(2), 282-286. https://doi.org/10.1037/a0014904

Sherman, G. D., Haidt, J., Iyer, R., \& Coan, J. A. (2013). Individual differences in the physical embodiment of care: Prosocially oriented women respond to cuteness by becoming more physically careful. Emotion, 13(1), 151-158. https://doi.org/10.1037/a0029259

Signal, T. D., \& Taylor, N. (2007). Attitude to animals and empathy: Comparing animal protection and general community samples. Anthrozoös, 20(2), 125-130.

Simmons, J. P., Nelson, L. D., \& Simonsohn, U. (2012). A 21 word solution. Available at SSRN 2160588. Retrieved from http://papers.ssrn.com/sol3/Papers.cfm?abstract_id=2160588

Sprengelmeyer, R., Perrett, D. I., Fagan, E. C., Cornwell, R. E., Lobmaier, J. S., Sprengelmeyer, A., ... Young, A. W. (2009). The cutest little baby face: a hormonal link to sensitivity to cuteness in infant faces. Psychological Science, 20(2), 149154. https://doi.org/10.1111/j.1467-9280.2009.02272.x

Steinnes, K. K. (2017). Too Cute for Words: Cuteness Evokes the Kama Muta Emotion and Motivates Communal Sharing (Master Thesis). University of Oslo, Oslo.

von Keyserlingk, M. A. G., Cestari, A. A., Franks, B., Fregonesi, J. A., \& Weary, D. M. (2017). Dairy cows value access to pasture as highly as fresh feed. Scientific Reports, 7 , srep44953. https://doi.org/10.1038/srep44953 
Tables

Table 1. Means and standard deviations of main measures in Study 1 presented for conditions separately. Comparisons across conditions are computed using a Welch's t-test.

\begin{tabular}{|c|c|c|c|c|c|c|c|}
\hline \multirow[t]{3}{*}{ Variable } & & & \multirow{3}{*}{$t$} & \multirow{3}{*}{$\mathrm{df}$} & \multirow{3}{*}{$p$} & \multirow{3}{*}{$d[95 \% \mathrm{CI}]$} & \multirow{3}{*}{$\mathrm{BF}_{10}$} \\
\hline & \multicolumn{2}{|c|}{ Condition $M(S D)$} & & & & & \\
\hline & $\begin{array}{l}\text { Animal } \\
(n=127)\end{array}$ & $\begin{array}{l}\text { Neutral } \\
(n=126)\end{array}$ & & & & & \\
\hline State Dissociation & $3.13(1.57)$ & $4.55(1.53)$ & 7.23 & 251 & $<.001$ & $.91[.65,1.17]$ & $1.72 \mathrm{E}^{10}$ \\
\hline Empathy & $4.84(1.66)$ & $3.74(1.87)$ & 4.94 & 251 & $<.001$ & $.62[.37, .87]$ & $6.25 \mathrm{E}^{4}$ \\
\hline Humanness & $2.86(2.00)$ & $2.10(1.58)$ & 3.33 & 251 & .001 & $.42[.17, .67]$ & 21.24 \\
\hline Cuteness & $4.01(.85)$ & $3.55(1.00)$ & 4.01 & 251 & $<.001$ & $.50[.25, .75]$ & 339.5 \\
\hline Willingness to eat & $45.80(31.22)$ & $62.10(34.20)$ & 3.96 & 251 & $<.001$ & $.50[.25, .75]$ & 1312.03 \\
\hline
\end{tabular}

Table 2. Means and standard deviations of main measures in Study 2 presented for condition separately. Comparisons across conditions are computed using a Welch's t-test.

\begin{tabular}{|c|c|c|c|c|c|c|c|}
\hline \multirow[t]{3}{*}{ Variable } & & & & & & & \\
\hline & \multicolumn{2}{|c|}{ Condition $M(S D)$} & \multirow[t]{2}{*}{$t$} & \multirow[t]{2}{*}{$\mathrm{df}$} & \multirow[t]{2}{*}{$p$} & \multirow[t]{2}{*}{$d[95 \% \mathrm{CI}]$} & \multirow[t]{2}{*}{$\mathrm{BF}_{10}$} \\
\hline & Cute $(n=206)$ & Neutral $(n=201)$ & & & & & \\
\hline Empathy & $4.84(1.78)$ & $4.42(1.96)$ & 2.27 & 404 & .023 & $.23[.03, .42]$ & 1.48 \\
\hline Cuteness & $4.05(.85)$ & $3.68(1.01)$ & 4.03 & 405 & $<.001$ & $.40[.20, .60]$ & 331 \\
\hline Willingness to eat & $42.31(32.95)$ & $46.33(35.57)$ & 1.18 & 405 & .238 & $.12[-.08, .31]$ & .23 \\
\hline Angriness & $2.06(1.19)$ & $2.89(1.36)$ & 6.55 & 404 & $<.001$ & $.65[.45, .85]$ & $7.10 \mathrm{E}^{7}$ \\
\hline
\end{tabular}


Table 3. Means and standard deviations of main measures in Study 3 a presented for condition separately. Comparisons across conditions are computed using a multilevel model with intercepts varying according to participant and animal type. Note. $n$ refers to number of cases, $B$ denotes the unstandardized coefficient.

\begin{tabular}{|c|c|c|c|c|c|c|}
\hline \multicolumn{7}{|l|}{ Variable } \\
\hline & \multicolumn{2}{|c|}{ Condition $M(S D)$} & \multirow[t]{2}{*}{$F$} & \multirow[t]{2}{*}{$\mathrm{df}$} & \multirow[t]{2}{*}{$p$} & $B[95 \% \mathrm{CI}]$ \\
\hline & $\begin{array}{l}\text { Cute } \\
(n=439)\end{array}$ & $\begin{array}{l}\text { Neutral } \\
(n=446) \\
\end{array}$ & & & & \\
\hline Empathy & $4.11(1.84)$ & $4.03(1.85)$ & 14.46 & 606.44 & $<.001$ & {$[.09, .27]$} \\
\hline Cuteness & $3.79(.91)$ & $3.36(1.05)$ & 95.91 & 693.88 & $<.001$ & {$[.38, .57]$} \\
\hline Willingness to eat & 49.08 (32.09) & $53.47(30.91)$ & 18.45 & 619.13 & $<.001$ & {$[-6.30,-2.35]$} \\
\hline Caretaking & $3.95(1.68)$ & $3.94(1.71)$ & 5.09 & 611.21 & .024 & {$[.01, .20]$} \\
\hline Angriness & $1.56(.92)$ & $2.04(1.24)$ & 67.65 & 764.35 & $<.001$ & {$[-.64,-.39]$} \\
\hline Perceived Age & $2.61(1.70)$ & $4.55(1.89)$ & 327.26 & 754.98 & $<.001$ & {$[-2.06,-1.66]$} \\
\hline
\end{tabular}

Table 4. Means and standard deviations of main measures in Study $3 b$ presented for condition separately. Comparisons across conditions are computed using a multilevel model with intercepts varying according to participant and animal type. Note. $n$ refers to number of cases , B denotes the unstandardized coefficient.

\begin{tabular}{|c|c|c|c|c|c|c|}
\hline \multirow[t]{3}{*}{ Variable } & & & & & & \\
\hline & \multicolumn{2}{|c|}{ Condition $M(S D)$} & \multirow[t]{2}{*}{$F$} & \multirow[t]{2}{*}{ df } & \multirow[t]{2}{*}{$p$} & \multirow[t]{2}{*}{$B[95 \% \mathrm{CI}]$} \\
\hline & $\begin{array}{l}\text { Cute } \\
(n=163)\end{array}$ & $\begin{array}{l}\text { Neutral } \\
(n=161)\end{array}$ & & & & \\
\hline Empathy & $4.20(1.57)$ & $3.87(1.53)$ & 20.76 & 223.49 & $<.001$ & $.32[.18, .46]$ \\
\hline Cuteness & $3.88(.84)$ & $3.43(.88)$ & 47.04 & 246.41 & $<.001$ & $.46[.33, .59]$ \\
\hline Willingness to eat & $55.8128 .12)$ & $55.58(30.09)$ & 1.39 & 225.94 & .241 & $-1.83[-4.90,1.24]$ \\
\hline Caretaking & $4.31(1.31)$ & $4.29(1.40)$ & 6.05 & 223.16 & .015 & $.14[.03, .26]$ \\
\hline Angriness & $1.35(.69)$ & $1.96(1.21)$ & 58.79 & 302.28 & $<.001$ & $-.69[-.87,-.51]$ \\
\hline Perceived Age & $2.05(1.08)$ & $3.86(1.63)$ & 199.66 & 276.84 & $<.001$ & $-1.91[-2.17,-1.64]$ \\
\hline
\end{tabular}


Figures

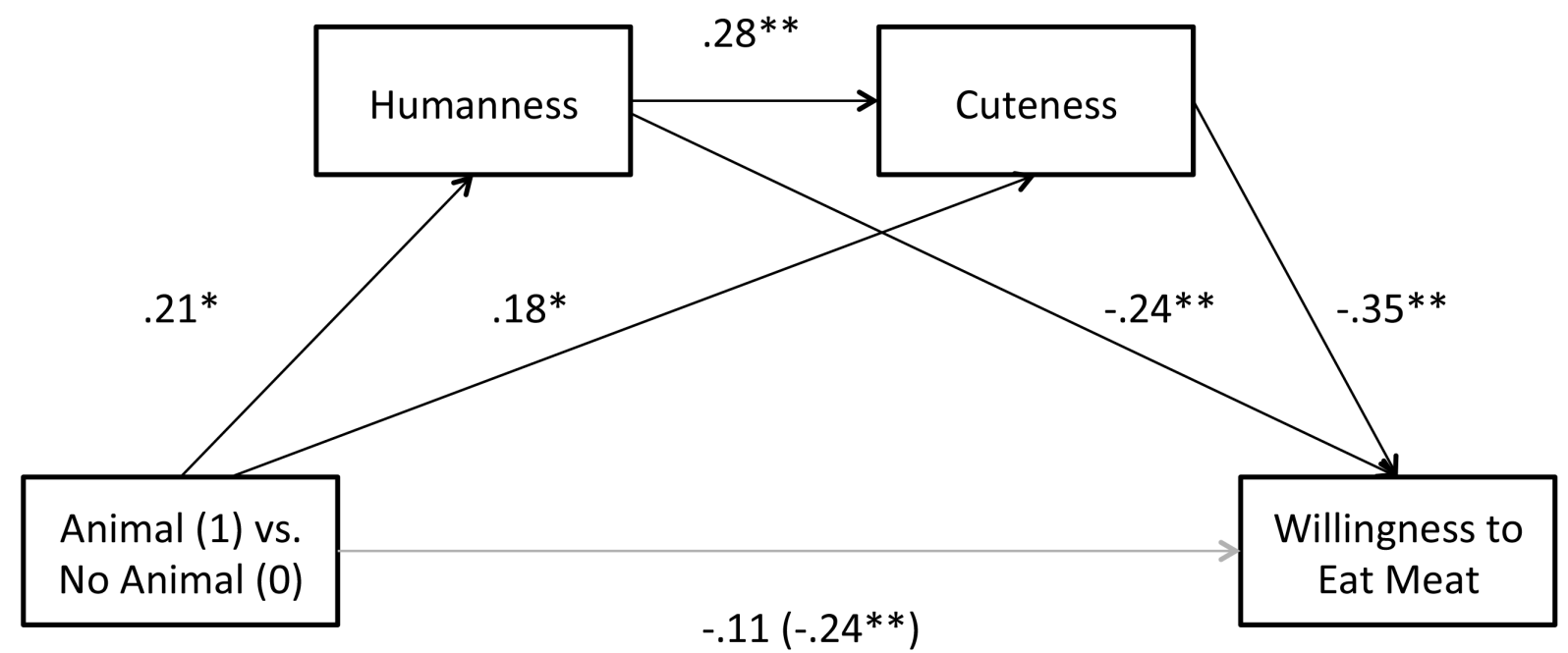

Figure 1. Humanness and cuteness fully mediated the effect of the experimental condition on willingness to eat the meat in Study 1. Standardized estimates are displayed. ${ }^{*} p<.05,{ }^{* *} p<$ .001 .

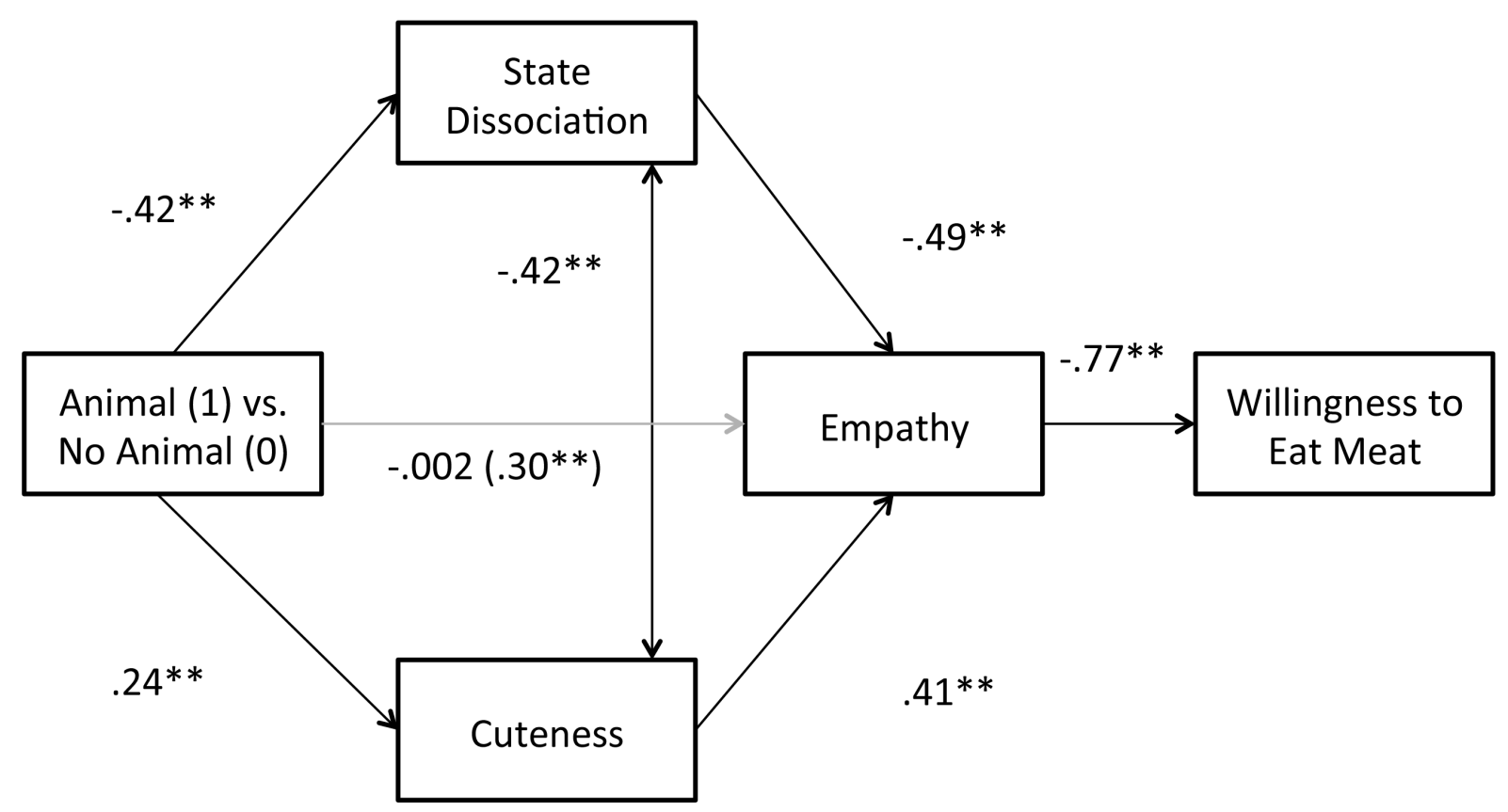

Figure 2. State dissociation and cuteness fully mediated the effect of the experimental condition on empathy in Study 1. Standardized estimates are displayed. ${ }^{*} p<.05,{ }^{*} p<.001$. 


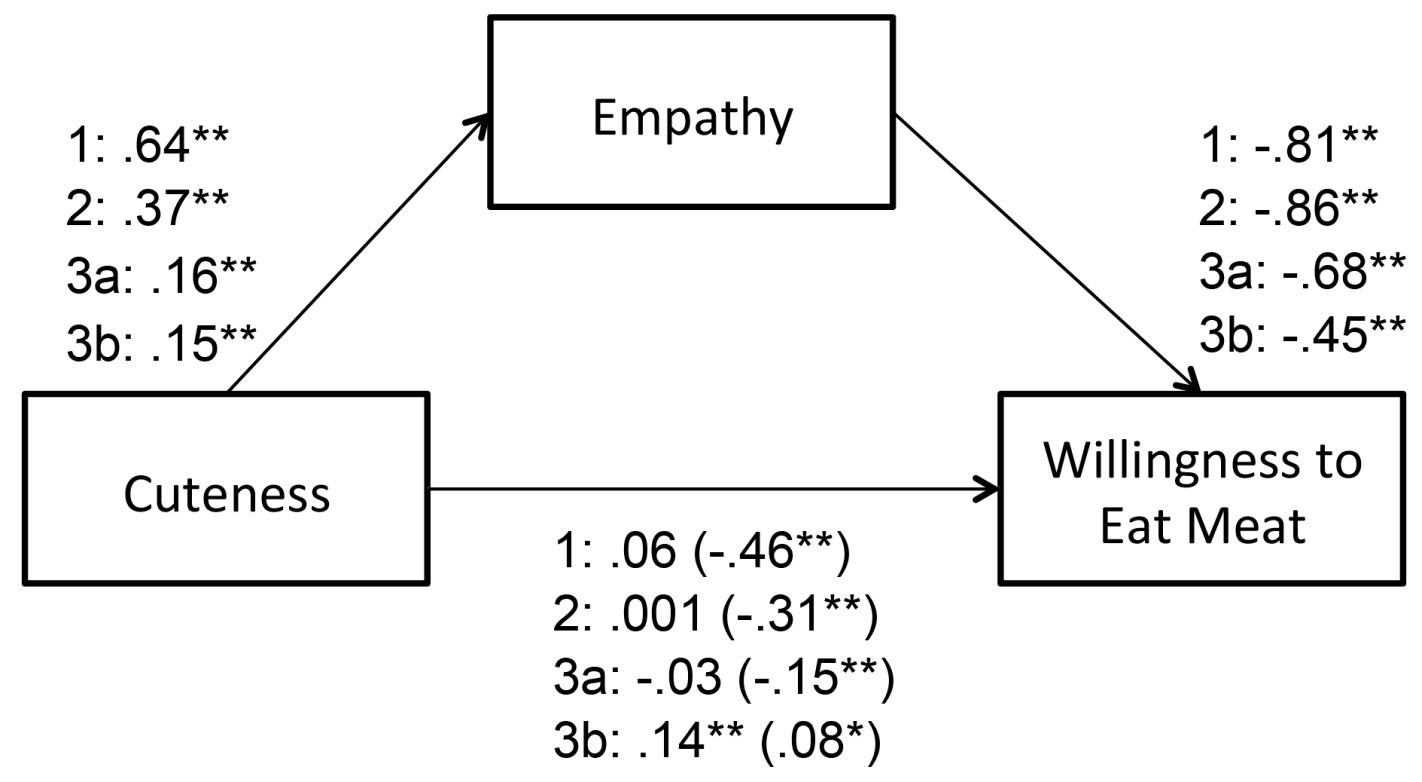

Figure 3. Empathy fully mediated the effect of cuteness on willingness to eat the meat in Study 1, 2, and 3a, and partially in Study 3b. Standardized estimates are displayed. ${ }^{*} p<.05$, $* * p<.001$.

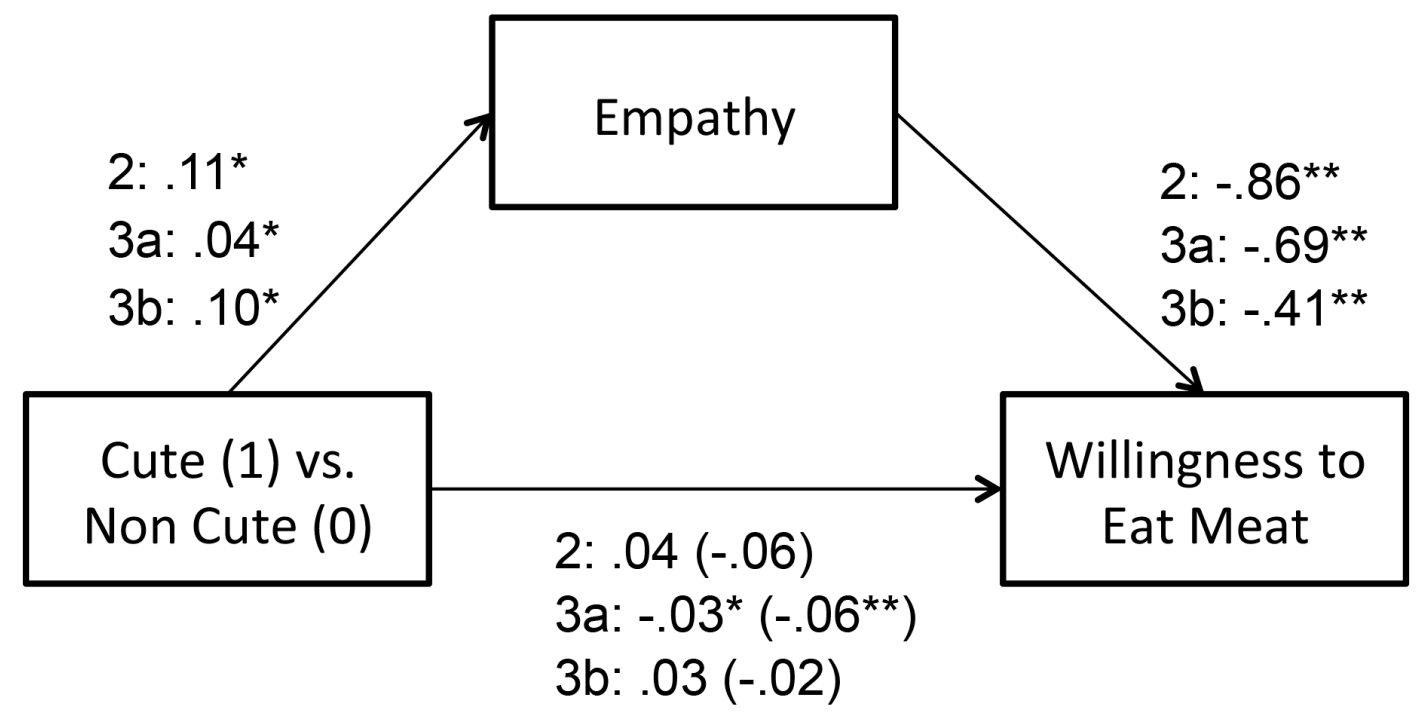

Figure 4. Empathy fully mediated the effect of the experimental condition on willingness to eat the meat in Study 2 and 3b, while partially in Study 3a. Standardized estimates are displayed. $* p<.05, * * p<.001$. 


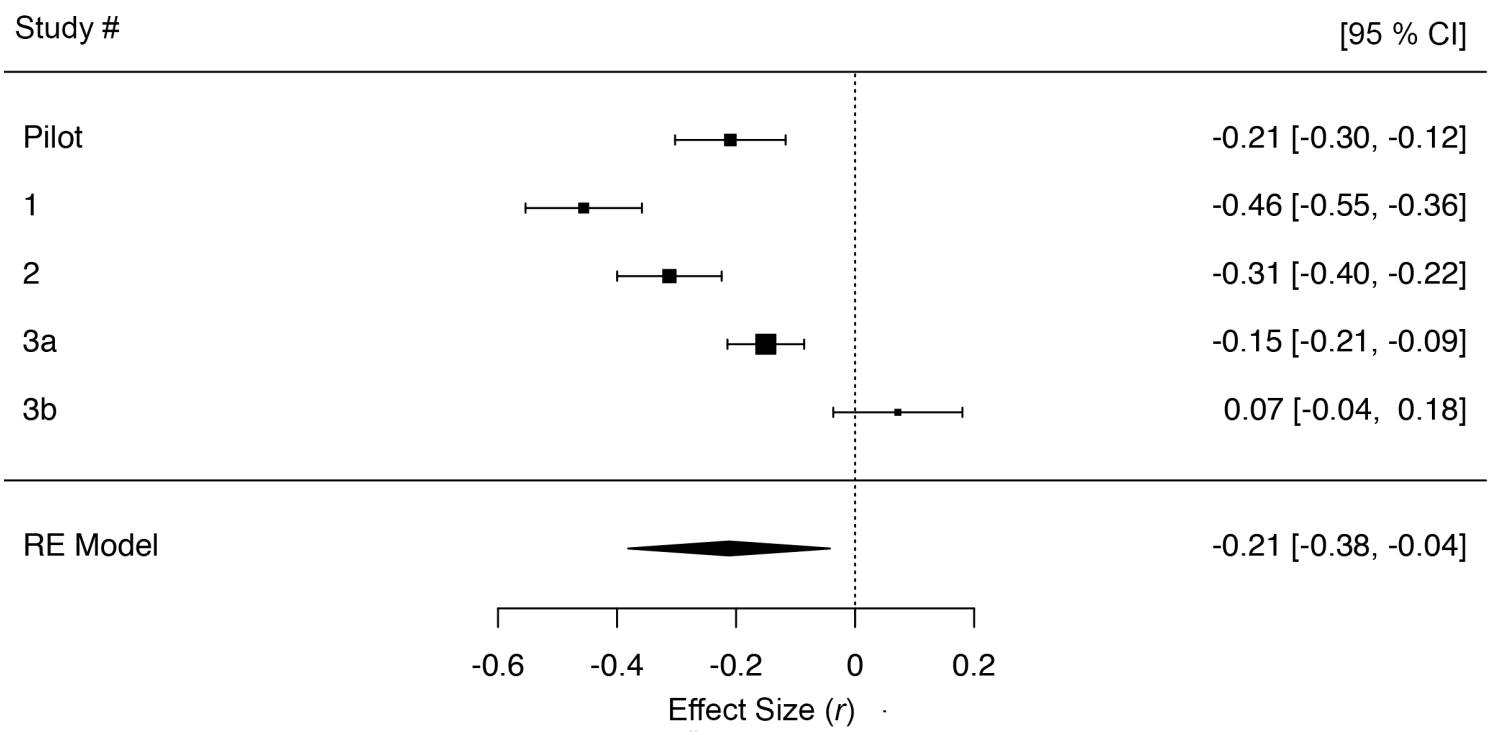

Figure 5. Random effects model of association between the cuteness rating and willingness to eat the meat across all four studies. Heterogeneity tests: $Q(4)=58.75, p<.001, I^{2}=94.61$ $[84.69,99.37]$.

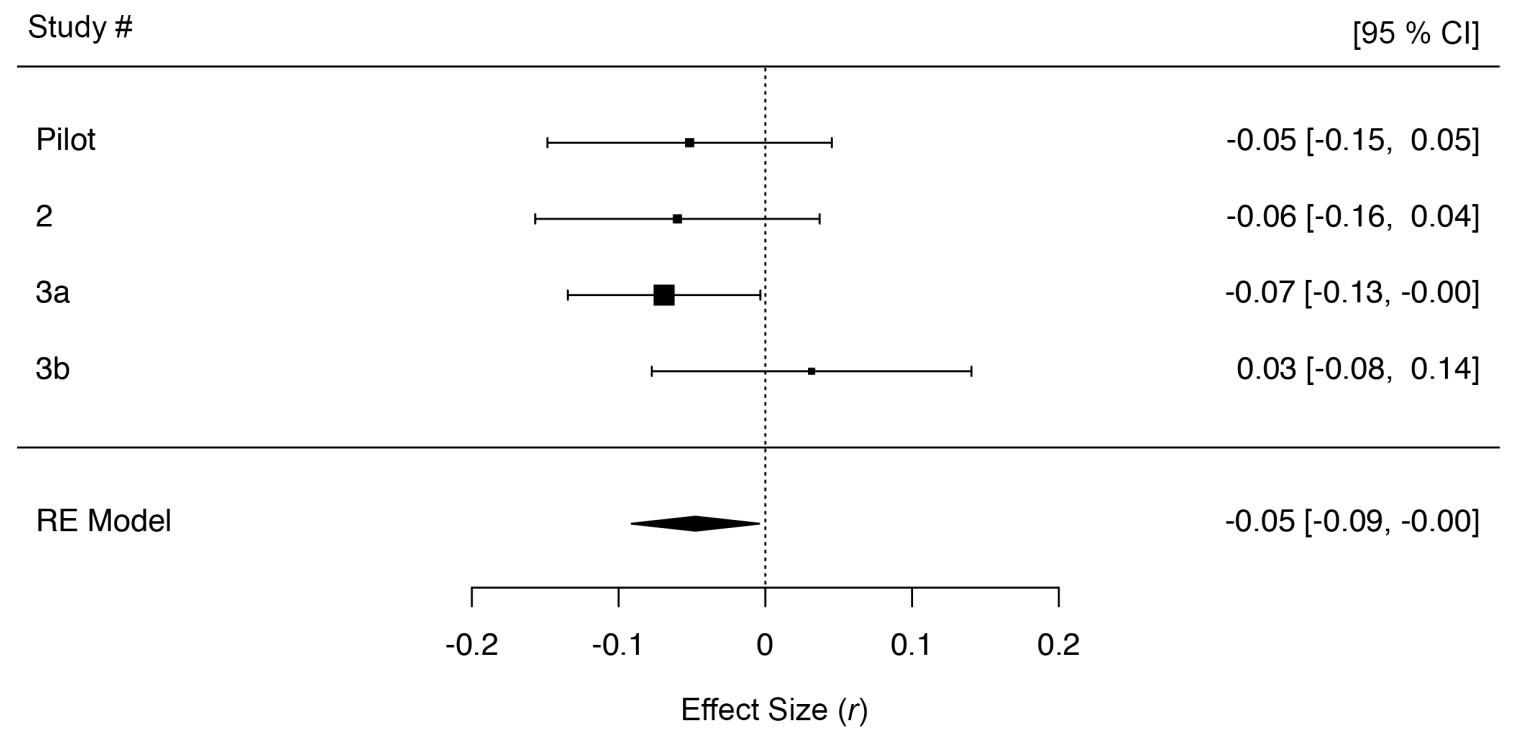

Figure 6. Random effects model of association between the experimental condition (cute vs. non-cute) and willingness to eat the meat across three studies. A negative correlation coefficient signifies that participants in the cute condition (1) indicated less willingness to eat the meat. Heterogeneity tests: $Q(3)=2.50, p=.47, I^{2}=0[0,92.83]$. 